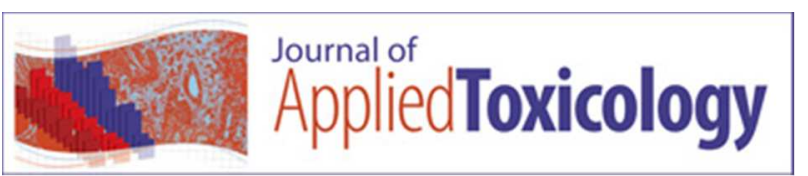

\title{
Relationship between increasing concentrations of two carcinogens and statistical image descriptors of foci morphology in the Cell Transformation Assay
}

\begin{tabular}{|c|c|}
\hline Journal: & Journal of Applied Toxicology \\
\hline Manuscript ID & JAT-16-0251.R2 \\
\hline Wiley - Manuscript type: & Research Article \\
\hline Date Submitted by the Author: & $\mathrm{n} / \mathrm{a}$ \\
\hline \multirow[t]{2}{*}{ Complete List of Authors: } & $\begin{array}{l}\text { Callegaro, Giulia; Universita degli Studi di Milano-Bicocca Dipartimento di } \\
\text { Scienze dell\'Ambiente e del Territorio e di Scienze della Terra } \\
\text { Corvi, Raffaella; European Commission Joint Research Centre Ispra Sector, } \\
\text { European Union Reference Laboratory for Alternatives to Animal Testing } \\
\text { (EURL ECVAM), Systems Toxicology Unit } \\
\text { Salovaara, Susan; European Commission Joint Research Centre Ispra } \\
\text { Sector, European Union Reference Laboratory for Alternatives to Animal } \\
\text { Testing (EURL ECVAM), Systems Toxicology Unit } \\
\text { Urani, Chiara; Universita degli Studi di Milano-Bicocca Dipartimento di } \\
\text { Scienze dell\'Ambiente e del Territorio e di Scienze della Terra, } \\
\text { Stefanini, Federico; Università di Firenze, Statistica, Informatica, } \\
\text { Applicazioni }\end{array}$ \\
\hline & $\begin{array}{l}\text { Cell Transformation Assay, BALB/c } 3 T 3 \text { cell line, Nickel chloride, Statistical } \\
\text { image descriptors, Dose-response, <i>Foci</i>, Benzo[a]Pyrene, } \\
\text { Extended linear mixed-effects models }\end{array}$ \\
\hline
\end{tabular}


1

2

3

4

5

6

7

8

9

10

\author{
1 Relationship between increasing concentrations of two carcinogens and statistical image \\ 2 descriptors of foci morphology in the Cell Transformation Assay \\ 3 \\ 4 \\ 5 \\ 6 \\ 720126 Milan, Italy \\ $8 \quad{ }^{2}$ European Union Reference Laboratory for Alternatives to Animal Testing (EURL ECVAM), Chemical \\ 9 Safety and Alternative Methods Unit; Directorate F, European Commission Joint Research Centre, TP 126, \\ 10 Via E. Fermi 2749, I-21027 Ispra (VA), Italy \\ $11{ }^{3}$ Department of Statistics, Computer Science, Applications, University of Florence - Viale Morgagni 59, \\ 1250100 Florence, Italy \\ 13 \\ 14 \\ 15
}

16

17

18

19

20

21

22

23

24

25

26

27

28

29

30

31

Running head: Effects of carcinogens concentration on foci morphology

Chiara Urani and Federico M. Stefanini are co-last Authors

\section{Corresponding Author:}

Federico Mattia Stefanini

Department of Statistics, Computer Science, Applications

University of Florence

Viale Morgagni 59, 50100 Florence, Italy

Phone: +390554237266

stefanini@disia.unifi.it 


\section{Abstract}

33 Cell transformation assays (CTAs) have long been proposed for the identification of chemical 34 carcinogenicity potential. The endpoint of these in vitro assays is represented by the phenotypic 35 alterations in cultured cells which are characterized by the change from the non-transformed to the transformed phenotype. Despite the wide fields of application and the numerous advantages of CTAs, their use in regulatory toxicology has been limited in part due to concerns about the subjective nature of visual scoring, i.e. the step in which transformed colonies or foci are evaluated through morphological features. An objective evaluation of morphological features has been previously obtained through automated digital processing of foci images to extract the value of three statistical image descriptors. In this study a further potential of the CTA using BALB/c 3T3 cells is addressed by analysing the effect of increasing concentrations of two known carcinogens with different mode of action (Benzo[a]Pyrene and $\mathrm{NiCl}_{2}$ ) on foci morphology.

The main result of our quantitative evaluation shows that the concentration of the considered carcinogens has an effect on foci morphology that is statistically significant for the mean of two among the three selected descriptors. Statistical significance also corresponds to visual relevance. The statistical analysis of variations in foci morphology due to concentration allowed to quantify morphological changes that can be visually appreciated but not precisely determined. Therefore, it has the potential of providing new quantitative parameters in CTAs, and of exploiting all the

\section{Short abstract for Table of Contents}


- Benzo[a]Pyrene

- Statistical image descriptors

- Dose-response

- Extended linear mixed-effects models

73 Abbreviations:

74 BD, Boundary Index

75 BIC, Bayesian Information Criteria

76 B[a]P, Benzo[a]Pyrene

$77 \mathrm{C}$, concentration

78 CTA, in vitro Cell Transformation Assay

79 ED, Equivalent Diameter

80 EFP, perimeter of the circle with equivalent area

81 FP, focus perimeter

82 MD, Median

83 PD, Petri dish 
84

85

86

87

88

89

90

91

92

93

94

95

96

97

98

99

100

101

102

103

104

105

106

107

108

109

110

111

112

113

114

115

116

117

118

\section{INTRODUCTION}

The cell transformation assays (CTAs) have long been proposed for the identification of chemical carcinogenicity potential. The endpoint of these in vitro assays is the induction of morphological alterations in cultured cells, which are characterized by the change from the non-transformed to the transformed phenotype. Long ago Barrett and Ts'o (1978) demonstrated that the study of oncogenesis in cell culture as a model for neoplastic transformation in vivo is strongly justified, and that the induction of specific morphological alterations in cultured cells reflects the features of cells exhibiting neoplastic potential in vivo.

These assays offer several advantages in comparison to the in vivo bioassays in rodents (OECD, TG451). They $i$ ) are faster and more cost efficient; $i$ ) allow the identification of not only genotoxic, but also of some non-genotoxic compounds; iii) may help to clarify in vitro genotoxic positive results; $i v$ ) provide a means to investigate tumor promotion activities and efficacy of chemopreventive agents; and v) support the 3Rs principles of replacement, reduction and refinement (EURL ECVAM, 2012). Current users of CTAs are the industry (chemical, agrochemical, pharmaceutical, and tobacco) for screening purposes, and academia mainly to investigate the molecular mechanisms of carcinogenesis. Although the use of CTAs is not currently an explicit regulatory requirement in any area of toxicology, their application is mentioned in different contexts in a number of guidance documents (EURL ECVAM Recommendation, 2013). In addition, two guidance documents on the CTA performed using Syrian hamster embryonic (SHE) cells and the Bhas 42 cell line, respectively, have been adopted by the Organization for Economic Cooperation and Development (OECD), which will probably stimulate the broader use of the assay in the future (OECD, 2015; 2016).

Despite the wide fields of applications and the numerous advantages of CTAs, their use in regulatory toxicology has been limited in part due to concerns about the reproducibility of the results between different laboratories in relation to the subjective nature of using morphological features for the identification of transformed cells, and the lack of understanding the mechanisms underlying the process of transformation (EURL ECVAM, 2012).

Recently several efforts have been undertaken to standardize and validate this test method, as well as to improve and refine different aspects of these assays. In particular, work is underway to respond to concerns that have been raised, such as the need for an objective scoring of transformed foci. This was addressed in the prevalidation and validation studies coordinated by the European Reference Laboratory on Alternative Methods (EURL ECVAM) and the Japanese Centre for the Validation of Alternative Methods (JaCVAM), which led to the publication of standardized 
119 procedure (Bohnenberger et al., 2012, Maire et al, 2012; Sasaki et al., 2012a; Sasaki et al., 2015; 120 OECD, 2016 Annex 2). In the framework of this study, a new statistical method to improve the 121 analysis of BALB/c 3T3 CTA data was developed (Hoffmann, 2012). Moreover, efforts addressed 122 at the automation of the visual scoring and of foci classification by means of image analysis are 123 ongoing in our group and several approaches based on extraction of statistical descriptors have 124 already been published (Ridder et al., 1997; Procaccianti et al., 2011; Urani et al., 2009, 2013; 125 Callegaro et al., 2015). In these studies, digital images of foci were acquired and regions of interest 126 corresponding to every single focus were identified. Statistical descriptors reflecting the coded 127 morphological features, which are at the basis of visual scoring of foci, were developed with the aim 128 of building a quantitative foci classifier.

129 The goal of this study is to provide the means to further exploit CTA results in order to consider the 130 information encoded in foci morphology. To this aim, the effect of different concentrations of two 131 known carcinogens, Benzo[a]Pyrene $(\mathrm{B}[\mathrm{a}] \mathrm{P})$ and nickel chloride $\left(\mathrm{NiCl}_{2}\right)$ on foci morphology is 132 assessed in the CTA using BALB/c 3T3, as captured by three selected foci descriptors. As far as we 133 are aware, this is the first attempt to quantitatively evaluate the role played by different 134 concentrations of carcinogens on the morphological endpoint in the transformation process. The 135 two carcinogens were selected based on their different modes of action, (B[a]P) which exerts its 136 effects through genotoxic mechanisms, and $\mathrm{NiCl}_{2}$ as it is known to act through indirect interactions 137 with DNA.

138 139

\section{MATERIALS AND METHODS}

140

\subsection{CTA experiments}

142 The BALB/c 3T3 CTA experiments were previously performed by EURL ECVAM within two 143 studies. The first study aimed at evaluating the transforming properties of different forms of nickel 144 (Salovaara et al., in preparation) and the other one assessed the transforming potential of B[a]P as 145 part of a broader study aimed at prevalidating the BALB/c 3T3 CTA (Corvi et al., 2012; Sasaki et 146 al., 2012b). The assays were carried out according to the experimental protocol used in the EURL 147 ECVAM prevalidation study (Corvi et al., 2012; Sasaki et al., 2012b). A concentration of $4 \mu \mathrm{g} / \mathrm{ml}$ 148 of 3-Methylcholanthrene (MCA, CAS number \#56-49-5) was chosen as the positive control as this 149 concentration was reported to induce positive transformation responses (Tanaka et al., 2012). In the 150 present work we focused on the dishes treated with different concentrations of $\mathrm{NiCl}_{2}(\mathrm{CAS}$ number $151 \quad \# 7718-54-9)$, more precisely the tested concentrations were 250, 275, 300, 350 and $400 \mu \mathrm{M}$. (CAS 152 number \#50-32-8) Tested concentrations for B[a]P (CAS number \#50-32-8), were 0.0005, 0.005, $1530.001,0.05,0.125,0.625,3.125,15 \mu \mathrm{g} / \mathrm{ml}$. 
154 As described in the recommended protocol and in the photo catalogue, the scoring of transformed

155 foci was performed under a stereomicroscope according to predefined criteria related to 156 morphological characteristics, which allowed to assign the foci into three categories: Type I, Type 157 II and Type III (Tanaka et al. 2012; Sasaki et al., 2012a). Only Type III foci were considered as 158 transformed, and thus scored as positive. According to the recommended protocol, a focus with a 159 diameter of less than $2 \mathrm{~mm}$ is too small to be considered as transformed.

160 Type I foci are small, non-invasive and weakly basophilic. Type II foci are multilayered, contain 161 basophilic spindle shape cells, and exhibit some cell piling up and criss-crossing, although to a 162 limited extent. Type III foci show dense multilayering (piling up) and display deep basophilic 163 spindle-shape cells, which differ from the non-transformed monolayer of background cells, that 164 shows contact-inhibition, and upon confluence is composed of flat, epithelial-like cells.

165

166

167

168

169

170

171

172

173

174

175

176

177

178

179

180

181

182

183

184

185

186

187

188

\subsection{Workflow}

The effect of different concentrations of two carcinogens on foci morphology was investigated through image analysis, as described by the workflow in Figure 1.

After performing the experiments (Box 1), foci images were acquired under a stereomicroscope equipped with a digital camera (Box 2), and statistical image descriptors were calculated for each focus (Box 3). A selection of three previously developed descriptors (Urani et al., 2013) was used to summarize the morphological features that are recognized as relevant in visual scoring (Sasaki et al., 2012a). As a result, a database comprising descriptors of foci morphology was built (Box 4), in which each collected focus is stored together with the value taken by its three descriptors, the carcinogen concentration and further auxiliary information represented by the label of the Petri dish in which the focus was acquired. The methodologies used in these steps, shown in Figure 1 by Boxes 2, 3, and 4, are detailed by Callegaro et al. (2015) and summarized in section 2.3. The effect of concentration on foci morphology, as captured by selected image descriptors, was studied by developing extended linear mixed-effects models (Pinheiro and Bates, 2000, p.202 eq. 5.1) in which the correlation existing among foci collected from the same Petri dish was also taken into consideration. In each final model ( 3 descriptors times 2 carcinogens $=6$ final models), a statistical test of the hypothesis stating the null effect of carcinogen concentration on foci morphology was performed. Further details about the statistical analysis in Boxes $5 \mathrm{~b}$ and $5 \mathrm{c}$ of Figure 1 are provided in section 2.4.

\subsection{Foci digitizing and data acquisition}

Foci images were acquired by adopting the procedure previously developed by Callegaro et al. (2015), and briefly described below. 
189 Acquisition was performed under a stereomicroscope (Zeiss, Stemi SV6) equipped with 6.3× lens

190 (Carl Zeiss, Arese, Italy) and a digital camera (AxioCam Mrc5, 36 bit). Images were saved in TIFF191 48bit RGB format and had a size of $2572 \times 1928$ pixels, where 1 pixel is equivalent to a real size of $1926.7842 \times 10^{-3} \mathrm{~mm}(1 \mathrm{~cm}=1474$ pixels $)$. Only fully transformed Type III foci were considered for the 193 acquisition.

194 Foci images comprised both the region corresponding to the transformed focus and a fraction of the 195 surrounding monolayer of non-transformed cells. An original segmentation algorithm coded in 196 Python (Python Software Foundation) was developed by Callegaro et al. (2015) in order to isolate 197 the focus regions from the surrounding monolayer. The algorithm acquires a RGB colour space 198 focus image, converts it into the HSV colour space and separates the region corresponding to each 199 focus from the surrounding monolayer by setting appropriate intensity thresholds.

200 During the segmentation process a subset of all images (around $30 \%$ for $\mathrm{NiCl}_{2}$ dataset, around $40 \%$ 201 for B[a]P dataset) was discarded, mainly due to pen marks used in the visual scoring process. The 202 derived $\mathrm{NiCl}_{2}$ dataset comprised a collection of 165 Type III foci images, obtained from dishes 203 tested with all the five $\mathrm{NiCl}_{2}$ concentrations specified in section 2.1. While the $\mathrm{B}$ [a]P derived 204 dataset comprised 224 Type III foci images, obtained from dishes tested with all eight B[a]P 205 concentrations (see section 2.1).

206 The experiment has a balanced layout as regards the number of Petri dishes: ten Petri dishes were 207 available for each treatment (i.e. $\mathrm{NiCl}_{2}$ or $\mathrm{B}[\mathrm{a}] \mathrm{P}$ concentration). Nevertheless, a different number of 208 Type III foci was observed in Petri dishes treated at different concentrations of the same carcinogen, 209 therefore the resulting sample size is not constant over concentrations. The change in the number of 210 segmented foci at each concentration was also due to the pen marks on Petri dishes, but no 211 appreciable changes in the trend of number of foci depending on concentration are produced, as can 212 be seen in the graphs of Figure 2 and Figure S1. See Table 1, Table 2 and Figure 2 for details on the 213 structure of the two datasets after segmentation. Additional information about the number of foci 214 observed at different concentrations before segmentation is shown in Figure S1.

215 For each image region corresponding to a Type III focus included in the final database after 216 segmentation, three previously developed statistical descriptors were calculated (Urani et al., 2013). 217 These descriptors summarize three morphological features among those coded and visually scored 218 in BALB/c 3T3 CTA (Sasaki et al., 2012b), which are:

219 - Equivalent Diameter (ED);

220 - Median (MD);

221 - Boundary Index (BD).

222 The descriptor ED is defined as the diameter of the circle whose area is equal to the area of the 223 focus: $\mathrm{ED}=2(\sqrt{(\operatorname{area} / \pi)})$, where $\pi$ is the trigonometric constant. We selected the ED descriptor to 
224 evaluate the size of the focus, taking into account the foci's shape polymorphism.

225 The descriptor MD is the median of the distribution made by image grey-levels in the region 226 corresponding to a focus. The median value of this distribution depends on the degree of multilayer 227 growth. Given that the intensity of light source is constant, image grey-levels mostly depend on the 228 amount of light passing through the focus region: regions with dense piling up will correspond to 229 darker image pixels, while regions that are less multilayered will correspond to lighter pixels.

230 Finally, the BD descriptor takes high values when invasive growth in the surrounding monolayer is 231 pronounced, a typical feature of fully transformed cells belonging to Type III foci. Invasiveness 232 often implies boundary heterogeneity of foci, thus the BD index compares the actual perimeter of 233 the focus (FP) with the perimeter of the circle of equivalent area (EFP): $B D=\left(\frac{F P}{E F P}\right)-1$.

234 The above three descriptors were previously developed and applied to the analysis of foci images 235 obtained from the prevalidation study coordinated by EURL ECVAM (Tanaka et al., 2012; Urani et 236 al, 2013), which included a large amount of experiments conducted with coded and uncoded 237 chemicals.

\subsection{Statistical analysis}

240 A generic Type III focus in a given Petri dish $x_{P D}$ is characterized by the random variables $241 Y_{E D}, Y_{M D}, Y_{B D}$ describing the value taken by statistical descriptors ED, MD and BD. The joint 242 distribution of $Y_{E D}, Y_{M D}, Y_{B D}$ given the Petri dish $x_{P D}$ and the concentration $x_{C}$ was considered at the 243 start of model building. The factorization of the joint distribution function into univariate 244 conditional distributions also allowed to study the dependence existing among statistical 245 descriptors:

$$
\begin{aligned}
p\left(y_{E D}, y_{M D}, y_{B D} \mid x_{P D}, x_{C}, \theta\right)= & p\left(y_{E D} \mid, y_{M D}, y_{B D}, x_{P D}, x_{C}, \theta_{E D}\right) \cdot p\left(y_{M D} \mid y_{B D}, x_{P D}, x_{C}, \theta_{M D}\right) \cdot \\
& p\left(y_{B D} \mid x_{P D}, x_{C}, \theta_{B D}\right)
\end{aligned}
$$

where $\theta=\left(\theta_{E D}, \theta_{M D}, \theta_{B D}\right)^{T}$ is the partitioned vector of model parameters. In Figure 3 , the initial factorization for one single focus is shown by a Directed Acyclic Graph (details in Buntine, 1994) in which model parameters are omitted. Each conditional distribution on the right of equation (1) 251 was parameterized assuming a Normal response explained by conditioning variables. Thus the 252 starting model was in the class of linear mixed-effects models (Pinheiro and Bates, 2000) for each 253 statistical descriptor. The concentration values were rescaled in the range [1,3] for the $\mathrm{B}[\mathrm{a}] \mathrm{P}$ 254 carcinogen. The class of investigated extended linear mixed-effects models included linear 255 predictors made by low degree polynomials of explanatory variables and their interactions, while 256 the variance of the response was modelled as a function of the (rescaled) concentration. Model 257 fitting was performed by Maximum Likelihood, after considering foci as conditionally 258 exchangeable. A preliminary selection of reasonable models was performed looking for models 
259 with a small value of the Bayesian Information Criterion (BIC), while a Likelihood ratio test (Cox 260 and Hinkley, 1974) for the effect of concentration was performed by comparing models 261 with/without the explanatory variable concentration.

262 The final model of each descriptor belongs to the class of extended linear models (Pinheiro and 263 Bates, 2000, p.202 eq. 5.1). The within group correlation existing among values of a descriptor 264 assessed on foci located in the same Petri dish was computed by exploiting an $\mathrm{R}$ object in the class 265 corCompSymm (nlme package, Pinheiro et al., 2015): we considered the Petri dishes as separate 266 environments, characterised by micro-fluctuations of experimental conditions shared by all foci in 267 each dish.

268 Residuals calculated from the final model of each image descriptor were graphically inspected to 269 check model assumptions. Details about model diagnostic are provided as Supporting Information, 270 e.g. quantile-quantile plots of residuals for each model (Figures S2-S3).

271 All statistical computations were performed in R (R Core Team, 2012), using nlme (Pinheiro et al., 272 2015) and effects packages (Fox, 2003).

273

274

\subsubsection{The factorization of the joint distribution}

275 The statistical image descriptors ED, MD and BD carry information about morphological features 276 jointly used for foci scoring, as previously shown by Urani et al. (2013), therefore the correlation 277 among them was expected. The configuration of considered dependencies is represented in Figure 278 3. The blue directed arrows shown in the graph represent dependence between the considered 279 descriptors (details in Buntine, 1994). The influence of carcinogen concentration on the selected 280 statistical descriptors is represented by yellow directed arrows starting in node C (Figure 3), while 281 yellow arrows leaving node PD and reaching BD, ED and MD indicate the effect of Petri dish 282 environment on foci morphology.

283 The factorization shown in equation 1 (section 2.4.) was selected by taking into account the specific 284 features of each descriptor before looking at the data. A focus can expand in the x-y space of the 285 Petri dish, determining its size, but it also grows in the third direction, the $\mathrm{z}$ axis of the Petri dish. 286 Hence, it is expected that the MD descriptor would be also informative for the ED descriptor, which 287 describes the dimension of a focus. Furthermore, the ability to invade the surrounding monolayer of 288 non-transformed cells can be considered as influencing the growth process in the two-dimensional 289 space of the Petri dish. Accordingly, the dependence relationship between ED and BD descriptors 290 has to be taken into account (blue arrows in Figure 3).

291 Transformed BALB/c 3T3 cells in a focus exhibit different growth characteristics from those of 292 non-transformed cells of the surrounding monolayer, primarily represented by a loss of contact293 inhibition and uncontrolled proliferation due to complex molecular mechanisms. In a focus 
294 displaying these properties, the invasion on the monolayer of non-transformed cells can be 295 observed, often by means of prolonged arms and vortexes of polarized cells (see for example figure 296 7D of Urani et al., 2013). This behaviour of uncontrolled growth and invasiveness influences the in 297 vitro three-dimensional growth (multilayering) of foci. Thus we developed a model for the response 298 of the MD descriptor in which BD is an explanatory variable.

299 In addition, each Petri dish represents a separate environment in which foci are exposed to the same 300 micro-environmental fluctuations and experimental errors, in contrast to foci observed in different 301 dishes. For this reason, we have included into the model an intra-class correlation parameter 302 (Pinheiro and Bates, 2000) which describes the degree of resemblance of foci in the same Petri dish 303 for a given descriptor, as detailed in section 2.4. Note that, in the final model, we did not estimate 304 the effects of Petri dishes since not relevant for the estimate of the effect of concentration on foci 305 morphology, thus reducing the number of model unknowns.

306

307 2.4.2. Models

308 The estimated expected values of the three conditional distributions for the $\mathrm{NiCl}_{2}$ and $\mathrm{B}[\mathrm{a}] \mathrm{P}$ 309 carcinogens are shown respectively in Table 3 and 4, just before testing for the contribution of 310 concentration, therefore the concentration is included as explanatory variable. 


\section{RESULTS}

\subsection{Conditional models of statistical descriptors given the concentration of a carcinogen} Considering the factorization of the equation 1 and represented in Figure 3, in this section the final models of each image descriptor given a specific carcinogen are described and commented. For details about model fitting, see sections 2.4.1 and 2.4.2.

\subsubsection{The Equivalent Diameter does not depend on carcinogen concentration}

Models built to test the dependence of the expected value of ED on carcinogen concentration are indicated as model $\mathrm{N} 1\left(\mathrm{NiCl}_{2}\right.$ dataset) and model $\mathrm{B} 1(\mathrm{~B}[\mathrm{a}] \mathrm{P}$ dataset $)$ and they both include the other two descriptors, $\mathrm{MD}$ and $\mathrm{BD}$, as explanatory variables. The model equations are shown in Table 3 and 4, first row. A remarkable feature of both models is that the effect of concentration on ED after including MD and BD was not found statistically significant. For model N1, the likelihood ratio test did not lead to the rejection of the hypotheses stating the null effect of concentration, with a p-value equal to 0.2427 . Likewise, for model B1, the likelihood ratio test did not lead to the rejection of the hypothesis stating the null effect of concentration, with a p-value equal to 0.8781 . It is worth noticing that, although the expected value of ED does not depend on concentration, the value of variance does. Therefore, the conditional distribution of ED of both models indeed depends on concentration.

In practical terms, the average size of a focus does not depend on the concentration of a carcinogen when the comparison is performed among foc $i$ with the same values of BD and of MD. Graphical summaries from the fitted models are shown in Figure 4, panels $\mathrm{C}$ and $\mathrm{H}$, where linear relationships between the focus size and the concentration of the treatment can be observed. While the expected values for model $\mathrm{B} 1$ vary with $\mathrm{B}[\mathrm{a}] \mathrm{P}$ concentration according to a straight line whose slope is close to zero (panel $\mathrm{H})$, for model $\mathrm{N} 1\left(\mathrm{NiCl}_{2}\right.$ carcinogen) the correspondent slope is negative instead (panel C). Still, testing for the partial contribution exerted by concentration as explanatory variable led to the conclusion that concentration is not significant, as suggested also by the overlapping confidence intervals in Figure 4H. For each model, four examples of foci are provided in the same figure, whose values of ED can be found in the extreme regions of the graph: for model N1 in Figure 4, panels A, B, D and E, for model B1 in the same Figure, panels F, G, I, J. Regardless the carcinogen concentration, small foci having low values for the ED descriptor (Figures 4, panels $\mathrm{A}$ and $\mathrm{D}, \mathrm{F}$ and $\mathrm{G}$ ) or bigger foci showing higher values for the ED descriptor (Figure 4, panels B and E, I and J) can be observed.

The dependence relationship between ED and MD descriptors was further explored by elaboration from fitted models. Considering the models presented here (see Table 3 and 4), a general large focus 
346 is characterized by a substantial ability to grow into multilayers, as shown by the focus in Figure 4, 347 panels B and E, G and J. A reduced multilayer growth, in contrast, can be found in small foci, as the 348 four provided in Figure 4, panels A and D, F and I.

349 Keeping in mind that MD is directly proportional to the amount of light in the focus grey-level 350 image, low values for the MD descriptor will correspond to darker foci, thus foci characterised by 351 several layers of cells. While high values for the MD descriptor will be associated with less 352 multilayered foci.

353 Indeed, an inverse relationship can be found plotting the fitted values of ED versus MD descriptor, 354 as provided in Supporting Information, Figure S2 and S3, panels G.

355 A remarkable result from our analyses is that we found the same qualitative relationships among 356 descriptors and of the concentration on descriptors, despite that models were fitted to data from two 357 different types of carcinogen, one genotoxic and one non-genotoxic. Thus it will be interesting in 358 the future to perform similar studies on additional carcinogens to test the general validity of such 359 qualitative features.

360

\subsubsection{The Median is dependent on carcinogen concentration}

362 Models built to test the dependence of the expected value of Median descriptor (MD) on carcinogen 363 concentration are indicated as Model N2 $\left(\mathrm{NiCl}_{2}\right.$ dataset) and Model B2 (B[a]P dataset) and they 364 both include BD as explanatory variable (see Figure 3). The model equations are shown in Table 3 365 and 4, second rows.

366 In both models, the partial contribution of concentration after including BD into the model is 367 statistically significant: for model N2, the likelihood ratio test for the hypothesis of no effect of 368 concentration was rejected with a p-value lower than 0.005 ( $\mathrm{p}$-value 0.0025$)$. Likewise, for model 369 B2, the likelihood ratio test for the hypothesis of no effect of concentration was rejected with a p370 value lower than 0.001 (p-value 0.0007). Hence for the average focus, multilayered growth changes 371 with the carcinogen concentration when the comparison is performed among foci characterized by 372 the same value of BD.

373 With $\mathrm{NiCl}_{2}$, the best model (N2) includes a second-degree polynomial in the concentration, thus the 374 change of MD's average is not linear with respect to the increase of concentration (Figure 5, panel 375 B).

376 Figure 5B describes the U-shaped non-linear behaviour of the MD descriptor obtained in Petri 377 dishes treated with increasing concentrations of $\mathrm{NiCl}_{2}$. A U-shaped trend can be observed with a 378 minimum located at about $310 \mu \mathrm{M}$.

379 As MD is directly proportional to the amount of light in the focus grey-level image, high values for 380 the MD descriptor will thus correspond to lighter foci, characterised by a reduced multilayering 
381 (Figure 5C). It follows that low values for the MD descriptor will be associated to foci characterized 382 by a significant multilayer growth, as the one presented in Figure 5D.

383 In the bounded range of considered concentrations, a first relative maximum, corresponding to 250 $384 \mu \mathrm{M} \mathrm{NiCl}_{2}$, is related to medium MD values, corresponding to foci not markedly and/or uniformly 385 multilayered (see as example focus in Figure 5A); moderately higher MD values can be observed corresponding to the highest concentration $\left(400 \mu \mathrm{M} \mathrm{NiCl}_{2}\right)$, as illustrated by the focus in Figure 5C.

387 In between, corresponding to intermediate $\mathrm{NiCl}_{2}$ concentrations $(275-350 \mu \mathrm{M})$, the absolute 388 minimum of the curve plotted in Figure 5B is located, suggesting the presence of multilayered foci 389 (as e.g. Figure 5D).

390 The best model for MD is not monotonic with the increase of concentration: higher and lower 391 concentrations induce the formation of foci displaying a reduced multilayer growth, while foci 392 obtained from intermediate $\mathrm{NiCl}_{2}$ concentrations show an increase in this feature.

393 By contrast, in the best model built for $\mathrm{B}[\mathrm{a}] \mathrm{P}$, the linear predictor is a straight line with respect to 394 the concentration (Figure 5, panel F). Considering again that MD is directly proportional to the amount of light in the focus grey-level image, at low $\mathrm{B}[\mathrm{a}] \mathrm{P}$ concentrations foci having small $\mathrm{MD}$ values are found (Figure 5E), in contrast to the higher MD values observed in foci at the highest 397 concentration.

398 Even if the two models differ in the shape of the curve representing the expected values in relation 399 to the concentrations, they partially share a similar feature, which is that the expected value of MD 400 is high at very high concentrations of carcinogens.

401 We conjecture that the trend at very high concentrations, shared by both carcinogens, could be due 402 to a combination of three factors. Firstly, these very high concentrations might have cytotoxic effects, dampening the multilayer growth of the transformed foci in these Petri dishes. This conclusion is supported by the fact that these Petri dishes had fewer foci, when compared to lower doses (Tables 1 and 2, Figure 2). Secondly, in foci induced by the highest concentrations used, a remarkable heterogeneity, a hallmark of both intermediate and mixed foci, was found as shown in Figure 5G. The MD descriptor applied to such a variable region, could have underestimated the overall multilayer growth of foci found in Petri dishes treated with high doses. From the statistical

412 The expected value of MD with respect to the concentration differs in the two models, in particular 413 in the low range of concentration, which could be due to specific differences of the carcinogens 414 mode of action. Nonetheless, the concentration range of $\mathrm{B}[\mathrm{a}] \mathrm{P}$ was experimentally designed to be 415 equally spaced on the logarithmic scale, hence a quite large part of the inner range has no 
416 observations on the original scale by design. Thus, there is a certain degree of uncertainty related to

417 the estimate at intermediate range of concentrations, therefore the apparent linear shape could not

418 be confirmed in specifically designed experiments with more distinct concentrations. Still, the 419 concentration exerts a statistically significant effect on the MD descriptor.

420 In Figures S2 and S3, further elaborations based on the fitted models (Model N2 and Model B2) 421 show the dependence relationship of MD from BD, in particular a monotonic relationship was 422 found in both cases. From these models, we expect that highly multilayered foci will be less 423 invasive than less multilayered foci.

424

425

426

\subsubsection{The Boundary Index is dependent on carcinogen concentration}

Models built to test the dependence of Boundary Index (BD) on carcinogen concentration are indicated as Model N3 and Model B3, respectively for $\mathrm{NiCl}_{2}$ and $\mathrm{B}[\mathrm{a}] \mathrm{P}$ datasets. The effect of concentration on BD was statistically significant in both models, and the expected value of BD is linear in the concentration (see Figures $6 \mathrm{~B}$ and $6 \mathrm{E}$ ): high values of $\mathrm{BD}$ result from high concentrations. In particular, for model N3, the likelihood ratio test for the hypothesis of no effect of concentration was rejected with a p-value lower than 0.001 (p-value 0.0004 ), while for model $\mathrm{B} 3$, the p-value of the test was lower than 0.0001 .

It is noteworthy to remember that the $\mathrm{BD}$ index is related to the degree of departure of the focus shape from that of a perfect circle with an equivalent area. Finger-like protuberances can be seen at the edge of the focus, as a result of the criss-cross growth at its edges (Sasaki et al., 2012a). The BD descriptor captures a trait of invasive growth that is characterized by heterogeneous growth at a focus boundary (Urani et al., 2013), therefore at higher concentrations of carcinogen more pronounced finger-like protuberances of foci are expected. 0.0005 to $3.125 \mu \mathrm{g} / \mathrm{ml}$ of $\mathrm{B}[\mathrm{a}] \mathrm{P})$, as described in section 3.1 .2 , fairly multilayered foci can be Figures 6A and 6D).

At higher concentrations, 350-400 $\mu \mathrm{M} \mathrm{NiCl}_{2}$ and $15 \mu \mathrm{g} / \mathrm{ml}$ of $\mathrm{B}[\mathrm{a}] \mathrm{P}$, foci showing a more

447 We remark, once more, that despite the different nature of the two carcinogens, qualitatively similar models for BD as a function of concentration were obtained. 


\section{DISCUSSION}

In a previous study (Urani et al., 2013) three statistical descriptors of foci morphology were developed with the aim of mimicking those features that are assessed in visual scoring in CTAs for chemical carcinogenicity testing. In this work, we selected $\mathrm{NiCl}_{2}$ and $\mathrm{B}[\mathrm{a}] \mathrm{P}$ foci images from previously performed CTAs, and we developed statistical models to test the effect of concentration on morphology. The joint distribution of all three statistical descriptors (ED, MD and BD) was factored into the product of three conditional distributions, after recognizing the statistical dependences existing among descriptors. Furthermore, we also considered that foci in the same Petri dish could be much more similar than foci located in different Petri dishes, due to the shared micro-environment.

This study suggests that the statistically significant effects of carcinogen concentration on some features of foci morphology, as represented by two of the three selected descriptors, are also coupled to visual relevance. Foci images obtained by testing two carcinogens with different mode of action were analyzed with the same method, and similar results in the structure of the dependencies with the concentration were obtained. Indeed, by inspecting the morphology of the foci obtained for each carcinogen at different concentrations, an appreciable degree of visual dissimilarity has been observed. At lower concentrations of both carcinogens $\left(250-300 \mu \mathrm{M} \mathrm{NiCl}_{2}\right.$ and $0.0005-3.125 \mu \mathrm{g} / \mathrm{ml} \mathrm{B}[\mathrm{a}] \mathrm{P})$ analyzed foci seem to grow on average in multilayers and in a circle-like shape. While at higher concentrations (300-400 $\mu \mathrm{M} \mathrm{NiCl}_{2}$ and $\left.15 \mu \mathrm{g} / \mathrm{ml} \mathrm{B}[\mathrm{a}] \mathrm{P}\right)$ the foci show a fragmented morphology characterized by less uniform multilayer growth. It must be pointed out that the ranges of $\mathrm{NiCl}_{2}$ and $\mathrm{B}[\mathrm{a}] \mathrm{P}$ were preliminarily selected by dose-range finding tests, as dictated by the CTA standard protocol, and qualitative similar shapes of boxplots for the number of foci in the concentration ranges were observed. By contrast, no clear differences can be seen in foci dimension in relation to the concentrations when the comparison is performed among foci with the same values of $\mathrm{BD}$ and of MD. In fact, as demonstrated by our statistical analyses, the concentration does not exert a direct effect on ED when MD and BD are already taken into account. In other terms, the expected value of ED does not change with concentration if foci of similar MD and $\mathrm{BD}$ are compared. It must be remarked that the carcinogen concentration has a significant effect on some aspects of foci morphology even if the concentration is included into the model as a qualitative factor (results not shown), that is without imposing a specific shape on the concentration-to-descriptor relationship. This is true for both the carcinogens considered. Note also that the variance of ED depends on the concentration, thus the variability changes with concentration.

In the models developed here, the dependence among descriptors and of a descriptor on concentration is not always linear. This may reflect the inherent biological complexity of the 
486 carcinogenic process. In particular, such non-linearity was evidenced in the $\mathrm{NiCl}_{2}$ model. Nickel 487 compounds and B[a]P are classified as Group I carcinogens (IARC, 2012a; IARC, 2012b) affecting 488 human health through occupational and environmental exposure. While $\mathrm{B}[\mathrm{a}] \mathrm{P}$ is known to act 489 through a genotoxic mechanism that involves biotransformation to highly reactive metabolites that 490 form covalent adducts to DNA and other genotoxic effects, carcinogenicity induced by nickel 491 compounds is characterized by the induction of oxidative stress through generation of reactive 492 oxygen species and by the interference with DNA repair pathways, thus leading to genetic 493 instability. Furthermore, nickel interferes with DNA methylation and histones acetylation, and 494 activates hypoxic signaling. These mechanisms taken together cause deregulation of cell 495 proliferation (Cameron et al., 2011; Sun et al., 2013; Magaye et al., 2012). As suggested by Haber 496 et al. (2000), the indirect interaction with DNA in soluble nickel-induced in vitro transformation 497 could imply a non-linear dose-response relationship. However, the authors also remarked that the 498 overall available data are insufficient to determine the doses at which such non-linearity occurs. 499 Nonetheless, they also underline that the suggestion of such non-linearity is consistent with the 500 negative animal carcinogenicity studies for soluble nickel, despite this negative results could also be 501 due to a different bioavailability and clearance of nickel ions after exposure to soluble nickel 502 compounds, as recently published (Goodman et al., 2011). As far as we are aware, no major 503 developments have occurred in this direction. In our dataset the effect of $\mathrm{NiCl}_{2}$ on one image 504 descriptor (MD) was here estimated to be non-linear as a function of concentration. Widening the 505 study of statistical descriptors to a broader concentration range might support the non-linearity 506 hypothesized by Haber et al. (2000), and in such expanded range they could be studied in a 507 quantitative way, thus possibly defining the concentration range at which non-linearity occurs.

508 In vitro cell transformation is considered to be a result of stepwise genotypic alterations, which 509 underlie the corresponding phenotypical ones (Smets, 1980; Keshava, 2000). Increasing 510 concentrations of carcinogens may lead to an accumulation of genetic changes (or different steps of 511 the global process of transformation), resulting in a variety of phenotypes which reflect different 512 molecular alterations. Quantitative morphological descriptors discriminate between the variety of 513 morphologies that can be visually appreciated but not precisely determined, leading to a detailed 514 evaluation of transformed foci. This may be especially useful to reduce the uncertainty in the 515 classification of mixed or intermediate foci.

516 Our analysis showed for the first time the dependence of Type III foci morphology on 517 concentration, thus it enabled the possibility of further distinctions inside the usual classification 518 scheme, as previously suggested by other authors (Keshava, 2000; Lu et al., 1986). The utility of 519 these detectable differences needs further investigations. Specific experiments could be designed to 520 evaluate a potential correspondence between different morphologies in Type III class foci and 
different tumor formation probabilities. Similarly, the molecular basis of observed differences within Type III foci should be further characterized to determine, for example, whether differences due to concentration detected by image descriptors are associated to specific molecular/metabolic signatures. Ongoing research points towards the molecular comprehension of in vitro cell transformation (Forcella et al., 2016; Priya et al., 2013; Ao et al., 2010; Rohrbeck et al., 2010; Walsh et al., 2009; Clemens et al., 2005). The potential availability of quantitative morphological markers representative in transformed cells of specific molecular pathways and specific classes of damages as a function of concentration could open the way towards an improvement of CTA.

Further applicative relevance of our approach includes the possibility to predict the values of the three descriptors summarizing the morphological features of foci in the CTA for other concentrations comprised in the tested range but not actually experimentally assessed.

Additional improvements of the proposed method can be envisaged. The fraction of all foci images lost in the segmentation step could be reduced in part by specific experimental precautions, for instance by limiting the use of pen marks outside the area of foci. In addition, improvements of the proposed descriptors might extract further quantitative information which is possibly relevant for the assessment of transformed foci. For example, the proposed Boundary Index (BD) captures just one of the aspects of invasive growth, in particular the degree of departure of the focus shape from that of an exact circle. Low values for the BD index seem to be related to foci composed of separated regions of cellular aggregates, rather than a unique focus body. A descriptor taking into account the heterogeneity within the same focus could improve both the assessment of invasiveness and the characterization of foci with mixed or intermediate morphology. Finally, large screening tests are needed for a detailed characterization of the effect of concentration on foci morphology, given that we provided positive evidence for just one compound for each major class of carcinogens (genotoxic and non-genotoxic).

The method presented in this study and applied to the BALB/c 3T3 CTA, could be potentially useful also in the CTA with Bhas 42 cells, for which an OECD Guidance Document has recently been published to support its use in regulatory applications (OECD, 2016, Sakai et al., 2011). The Bhas 42 cell line is in fact derived from BALB/c $3 \mathrm{~T} 3$ cells transfected with the $v$-Ha-ras protooncogene, consequently its transformed foci are characterized by the same morphological features as the BALB/c $3 \mathrm{~T} 3$ foci.

In conclusion, our quantitative evaluations mainly show that the concentration of the considered carcinogens exerts an effect on foci morphology. We believe that statistical descriptors of foci morphology have the potential of mimicking trained human scorers very well, but with the advantage of being both objective and quantitative. 
556 more reliable in vitro procedures when assessing carcinogenicity through an integrated testing

557 strategy, either in a full probabilistic (Stefanini, 2013) or a Weight of Evidence approach (ECHA

558 2010).

559

560

561

Acknowledgments

562 This work is partially funded by the University of Milan Bicocca (2014-ATE-0043 to CU) and by

563 the University of Florence (to FMS), funding framework "Strategic project of basic research, year

564 2015." The authors acknowledge the European Commission.

565

\section{REFERENCES}

567 Ao L, Liu JY, Liu WB, Gao LH, Hu R, Fang ZJ, Zhen ZX, Huang MH, Yang MS, Cao J. 2010.

568 Comparison of gene expression profiles in BALB/c 3T3 transformed foci exposed to tumor 569 promoting agents. Toxicol. In Vitro. 24: 430-438.

570 Barrett JC and Ts'o PO. 1978. Evidence for the progressive nature of neoplastic transformation in $571 \quad$ vitro. PNAS. 75 (8): 3761-3765.

572 Bohnenberger S, Kunkelmann T, Perschbcher S, Poth A, Pant K, Bruce SW, Sly JE, Schwind KR.

573 2012. Photo catalogue for the classification of cell colonies in the Syrian hamster embryo

$574 \quad$ (SHE) Cell Transformation Assay at pH 6.7. Mutat. Res. 744 (1): 82-96.

575 Buntine WL. 1994. Operations for Learning with Graphical Models. J. Artif. Intell. Res. 22: 159576225 .

577 Callegaro G, Stefanini FM, Colacci A, Vaccari M, Urani C. 2015. An improved classification of 578 foci for carcinogenicity testing by statistical descriptors. Toxicol. In Vitro. 29 (7): 1839-50.

579 Cameron KS, Buchner V and Tchounwou PB. 2011. Exploring the Molecular Mechanisms of 580 Nickel-Induced Genotoxicity and Carcinogenicity: A Literature Review. Rev. Environ. $581 \quad$ Health. 2011, 26(2): 81-92.

582 Clemens F, Verma R, Ramnath J, and Landolph JR. 2005. Amplification of the Ect2 proto583 oncogene and over-expression of Ect2 mRNA and protein in nickel compound and 584 methylcholanthrene-transformed 10T1/2 mouse fibroblast cell lines. Toxicol. Appl. $585 \quad$ Pharmac. 206, 138-149.

586 Corvi R, Aardema MJ, Gribaldo L, Hayashi M, Hoffmann S, Schechtman L, and Vanparys P. 2012. 587 ECVAM prevalidation study on in vitro cell transformation assays: General outline and 588 conclusions of the study. Mutat. Res. Genet. Toxicol. Environ. Mutagen. 744 (1): 12-19.

589 Cox DR, Hinkley DV. 1974. Theoretical Statistics. Chapman and Hall.

590 ECHA, 2010. Practical guide 2: How to report weight of evidence. Available at 
591

592

593

594

595

596

597

598

599

600

601

602

603

604

605

606

607

608

609

610

611

612

613

614

615

616

617

618

619

620

621

622

623

624

625

https://echa.europa.eu/documents/10162/.../pg_report_weight_of_evidence_en.pdf

EURL ECVAM. 2013. EURL ECVAM Recommendation on the cell transformation assays based on the Bhas 42 cell line. Available at https://eurl-ecvam.jrc.ec.europa.eu/eurl-ecvamrecommendations/files-bhas/EURL_ECVAM_Recommendation_Bhas-CTA_2013.pdf

EURL ECVAM. 2012. EURL ECVAM Recommendation on three Cell Transformation Assays using Syrian Hamster Embryo Cells (SHE) and the BALB/c 3T3 Mouse Fibroblast Cell Line for In Vitro Carcinogenicity Testing. Available at https://eurl-ecvam.jrc.ec.europa.eu/eurlecvam-recommendations/EURL-ECVAM -Recommendation.pdf

Forcella M, Callegaro G, Melchioretto P, Gribaldo L, Frattini M, Stefanini FM, Fusi P, Urani C. 2016. Cadmium-transformed cell in the in vitro Cell Transformation Assay reveal different proliferative behaviours and activated pathways. Toxicol. in Vitro. 36: 71-80.

Fox J. 2003. Effect Displays in R for Generalised Linear Models. J. Stat. Softw. 8(15): 1-27.

Goodman JE, Prueitt RL, Thakali S, and Oller AR. 2011. The nickel ion bioavailability model of the carcinogenic potential of nickel-containing substances in the lung. Crit. Rev. Toxicol. 41(2): 142-174.

Haber LT, Erdreicht L, Diamond GL, Maier AM, Ratney R, Zhao Q, and Dourson ML. 2000. Hazard Identification and Dose Response of Inhaled Nickel-Soluble Salts. Regul. Toxicol. Pharmacol. 31: 210-230.

Hoffmann S, Hothorn LA, Edler L, Kleensang A, Suzuki M, Phrakonkham P, and Gerhard D. 2012. Two new approaches to improve the analysis of BALB/c 3T3 cell transformation assay data. Mutat. Res. 744: 36-41.

International Agency for Research on Cancer. 2012a. IARC Monograph: arsenic, metals, fibres, and dusts (volume $100 \mathrm{C}$ ). A review of human Carcinogens. Lyone, France.

International Agency for Research on Cancer. 2012b. IARC Monograph: chemical agents and related occupations (volume $100 \mathrm{~F}$ ). A review of human Carcinogens. Lyone, France.

Keshava N. 2000. Tumorigenicity of morphologically distinct transformed foci induced by 3methylcholanthrene in BALB/c-3T3 cells. Mutat. Res. 447 (2): 281-6.

Lu Y P, Lasne C, Chouroulinkov I. 1986. Use of an orthogonal design method to study two-stage chemical carcinogenesis in BALB/3T3 cells, Carcinogenesis 7: 893-898.

Magaye R, Zhao J, Bowman L, Ding M. 2012. Genotoxicity and carcinogenicity of cobalt-, nickeland copper-based nanoparticles. Exp. Ther. Med. 4(4):551-561.

Maire MA, Rast C, Vasseur P. 2012. Photo catalogue for the classification of cell colonies in the Syrian hamster embryo (SHE) Cell Transformation Assay at pH 7.0. Mutat. Res. 744(1): 97110 .

OECD 2016. Guidance Document on the In Vitro Bhas 42 Cell Transformation Assay. 
626

627

628

629

630

631

632

633

634

635

636

637

638

639

640

641

642

643

644

645

646

647

648

649

650

651

652

653

654

655

656

657

658

659

660

Series on Testing \& Assessment No. 231.

(https://www.oecd.org/env/ehs/testing/ENV_JM_MONO(2016)1.pdf).

OECD 2015. Guidance Document on the In Vitro Syrian Hamster Embryo (SHE) Cell Transformation Assay. Series on Testing \& Assessment No. 214. (http://www.oecd.org/env/ehs/testing/seriesontestingandassessmentpublicationsbynumber.ht $\underline{\mathrm{m}})$

OECD 2009. Test Guideline 451 - Carcinogenicity studies. OECD Guidelines for the Testing of Chemicals, OECD, Paris. (http://www.oecd-ilibrary.org/environment/test-no-451carcinogenicity-studies_9789264071186-en.)

Pinheiro JC, and Bates DM. 2000. Chapter 5: Extending the Basic Linear Mixed-Effects Model. In Mixed-Effects Models in S and S-PLUS. Springer: New York; 201-267.

Pinheiro JC, Bates DM, DebRoy S, Sarkar D, and R Core Team. 2015. nlme: Linear and Nonlinear Mixed Effects Models, R package version 3.1-120. $\underline{\text { http://CRAN.R- }}$ project.org/package $=$ nlme

Python Software Foundation, Python language reference, version 2.7. URL: https://www.python.org/.

Priya S, Nigam A, Bajpai P, Kumar S. 2013. Dysregulation of pathways involved in the processing of cancer and microenvironment information in MCA+TPA transformed C3H/10T1/2 cells. In Vitro Cell. Dev. Biol. Anim. 49: 295-305.

Procaccianti C, Stefanini FM, Urani C. 2011. The Cell Transformation Assay: Toward a statistical classification of mixed and intermediate foci images. ATLA. 39(1):23-36.

R Core Team. 2012. R: A language and environment for statistical computing. R Foundation for Statistical Computing, Vienna, Austria. ISBN 3-900051-07-0, URL http://www.Rproject.org/

Ridder GM, Stuard SB, Kerckaert GA, Cody DB, LeBoeuf LA, Isfort RJ. 1997. Computerized image analysis of morphologically transformed and nontransformed Syrian hamster embryo (SHE) cell colonies: application to objective SHE cell transformation assay scoring. Carcinogenesis. 18: 1965-1972.

Rohrbeck A, Salinas G, Maaser K, Linge J, Salovaara S, Corvi R, Borlak J. 2010. Toxicogenomics applied to in vitro carcinogenicity testing with Balb/c 3T3 cells revealed a gene signature predictive of chemical carcinogens. Toxicol. Sci. 118: 31-41.

Sakai A, Sasaki K, Hayashi K, Muramatsu D, Arai S, Endou N, Kuroda S, Poth A, Bohnenberger S, Kunkelmann T, Asakura M, Hirose H, Ishii N, Mizuhashi F, Kasamoto S, Nagai M, Pant K, Bruce SW, Sly JE, Yamazaki S, Umeda M, Tanaka N. 2011. An international validation study of a Bhas 42 cell transformation assay for the prediction of chemical carcinogenicity. 
661

662

663

664

665

666

667

668

669

670

671

672

673

674

675

676

677

678

679

680

681

682

683

684

685

686

687

688

689

690

Mutat. Res. 725(1-2):57-77.

Sasaki K, Umeda M, Sakai A, Yamazaki S, Tanaka N. 2015. Transformation assay in Bhas 42 cells: a model using initiated cells to study mechanisms of carcinogenesis and predict carcinogenic potential of chemicals. J. Environ. Sci. Health C. Environ. Carcinog. Ecotoxicol. Rev. 33:135.

Sasaki K, Bohnenberger S, Hayashi K, Kunkelmann T, Muramatsu D, Poth A, Sakai A, Salovaara S, Tanaka N, Thomas BC, and Umeda M. 2012a. Photo catalogue for the classification of foci in the BALB/c 3 T3 cell transformation assay. Mutat. Res. 744: 42-53.

Sasaki K, Bohnenberger S, Hayashi K, Kunkelmann T, Muramatsu D, Phrakonkham P, Poth A, Sakai A, Salovaara S, Tanaka N, Thomas BC, and Umeda M. 2012b. Recommended protocol for the BALB/c 3T3 cell transformation assay. Mutat. Res. 744: 30-35.

Smets LA. 1980. Cell transformation as a model for tumor induction and neoplastic growth. Biochim. Biophys. Acta. 605 (1): 93-111.

Stefanini, FM. 2013. Comment: Bayesian network integrated testing strategy and beyond. Altex 30, 386-390.

Sun,H, Shamy M, and Costa M. 2013. Nickel and Epigenetic Gene Silencing. Genes (Basel) 4, 583-595.

Tanaka N, Bohnenberger S, Kunkelmann T, Munaro B, Ponti J, Poth A, Sabbioni E, Sakai A, Salovaara S, Sasaki K, Thomas BC, Umeda M. 2012. Prevalidation study of the BALB/c $3 \mathrm{~T} 3$ cell transformation assay for assessment of carcinogenic potential of chemicals. Mutat. Res. Genet. Toxicol. Environ. Mutagen. 744(1): 20-29.

Urani C, Corvi R, Callegaro G, and Stefanini FM. 2013. Objective scoring of transformed foci in BALB/c 3 T3 cell transformation assay by statistical image descriptors. Toxicol. in Vitro $\mathbf{2 7}$ : 1905-1912.

Urani C, Stefanini FM, Bussinelli L, Melchioretto P, Crosta GF. 2009. Image analysis and automatic classification of transformed foci. J. Microsc. 234: 269-279.

Walsh MJ, Bruce SW, Pant K, Carmichael PL, Scott AD and Martin FL. 2009. Discrimination of a transformation phenotype in Syrian golden hamster embryo (SHE) cells using ATR-FTIR spectroscopy. Toxicology. 258(1):33-8. 
691

692

693

694

695

696

697

698

699

700

701

702

703

704

705

706

707

708

\section{TABLES}

Table 1. The number of dishes and foci included in the final database following the segmentation process are shown for each $\mathrm{NiCl}_{2}$ concentration. The initial number of dishes available for each concentration was 10 .

\begin{tabular}{ccccc}
\hline Dose $(\mu \mathrm{M})$ & $\begin{array}{c}\text { \# dishes containing } \\
\text { Type IIII } \text { foci at the } \\
\text { end of CTA }\end{array}$ & $\begin{array}{c}\text { \# dishes considered } \\
\text { after segmentation }\end{array}$ & $\begin{array}{c}\text { total \# of foci (III) } \\
\text { before segmentation }\end{array}$ & $\begin{array}{c}\text { \# foci (III) after } \\
\text { segmentation }\end{array}$
\end{tabular}

\begin{tabular}{lcccc}
\hline 250 & 7 & 4 & 10 & 6 \\
\hline 275 & 10 & 10 & 33 & 23 \\
\hline 300 & 10 & 10 & 73 & 40 \\
\hline 350 & 10 & 10 & 137 & 78 \\
\hline 400 & 9 & 9 & 29 & 18 \\
\hline Total & 46 & 43 & 282 & 165 \\
\hline
\end{tabular}

Table 2. The number of dishes and foci included in the final database following the segmentation process are shown for each $\mathrm{B}[\mathrm{a}] \mathrm{P}$ concentration. The initial number of dishes available for each concentration was 10 .

\begin{tabular}{|c|c|c|c|c|}
\hline Dose $(\mu \mathrm{g} / \mathrm{ml})$ & $\begin{array}{l}\text { \# dishes containing } \\
\text { Type IIII foci at the } \\
\text { end of CTA }\end{array}$ & $\begin{array}{l}\# \text { dishes considered } \\
\text { after segmentation }\end{array}$ & $\begin{array}{l}\text { total \# of foci (III) } \\
\text { before segmentation }\end{array}$ & $\begin{array}{l}\text { \# foci (III) after } \\
\text { segmentation }\end{array}$ \\
\hline
\end{tabular}

\begin{tabular}{lcccc}
\hline 0.0005 & 4 & 4 & 6 & 4 \\
\hline 0.001 & 2 & 2 & 2 & 2 \\
\hline 0.005 & 7 & 7 & 15 & 13 \\
\hline 0.05 & 10 & 10 & 70 & 38 \\
\hline 0.125 & 10 & 10 & 92 & 44 \\
\hline 0.625 & 10 & 10 & 111 & 73 \\
\hline 3.125 & 10 & 10 & 74 & 41 \\
\hline 15 & 8 & 7 & 12 & 9 \\
\hline Total & 46 & 43 & 382 & 224 \\
\hline
\end{tabular}

http://mc.manuscriptcentral.com/apptox 
709 Table 3. Point estimates from the $\mathbf{~ N i C l}_{2}$ dataset. Expected value functions of each univariate 710 conditional distribution for the final models including $\mathrm{NiCl}_{2}$ concentration before testing the null 711 hypothesis of no effect by means of likelihood ratio tests. The estimated value of intraclass 712 correlation parameter is indicated as $\hat{\rho}$. The variance was modelled as a function of the rescaled 713 concentration $\tilde{x}_{C}$. In particular, the estimated conditional variance function in all models is defined 714 by the following equation: $\sigma_{y}^{2}=\sigma^{2}\left(\tilde{x}_{C}\right)^{2 \delta}$, where $\mathrm{y}$ is the descriptor considered as a response 715 variable.

716 Note that $\widehat{Y}_{E D} \equiv E\left[Y_{E D} \mid y_{M D}, y_{B D}, x_{P D}, x_{C}, \theta_{E D}\right]$.

Expected value

Variance function

Correlation

\begin{tabular}{ccc}
\hline $\mathrm{N} 1: \hat{Y}_{E D}=51993.62-141843.64 y_{M D}+97223.63 y_{M D}^{2}+$ & $\hat{\sigma}=242.08$ & $\hat{\rho}=0.15$ \\
$+1437.82 y_{B D}-108.02 x_{C}-1848.83 y_{M D} y_{B D}$ & $\hat{\delta}=0.42$ & \\
\hline $\mathrm{N} 2: \hat{Y}_{M D}=0.72+0.013 y_{B D}-0.13 x_{C}+0.13 x_{C}^{2}$ & $\hat{\sigma}=0.024$ & $\hat{\rho}=0.01$ \\
& $\hat{\delta}=-0.16$ & \\
\hline $\mathrm{N} 3: \hat{Y}_{B D}=1.25+1.28 x_{C}$ & $\hat{\sigma}=0.94$ & $\hat{\rho}=0.006$
\end{tabular}

717

718

719

720

721

722

723

724

725

726

727 Note that $\widehat{Y}_{E D} \equiv E\left[Y_{E D} \mid y_{M D}, y_{B D}, x_{P D}, x_{C}, \theta_{E D}\right]$.

\begin{tabular}{|c|c|c|}
\hline Expected value & $\begin{array}{l}\text { Variance } \\
\text { function }\end{array}$ & Correlation \\
\hline B1: $\hat{Y}_{E D}=3232.74-4405.34 y_{M D}+247.31 y_{B D}-38.68 y_{B D}^{2}-0.81 x_{C}$ & $\begin{array}{c}\hat{\sigma}=228.70 \\
\hat{\delta}=-0.06\end{array}$ & $\hat{\rho}=0.15$ \\
\hline $\mathrm{B} 2: \hat{Y}_{M D}=0.67+0.003 y_{B D}+0.001 x_{C}$ & $\begin{array}{c}\hat{\sigma}=0.016 \\
\hat{\delta}=0.03\end{array}$ & $\hat{\rho}=0.06$ \\
\hline B3: $\widehat{Y}_{B D}=1.60+0.08 x_{C}$ & $\begin{array}{c}\hat{\sigma}=0.72 \\
\hat{\delta}=-0.06\end{array}$ & $\hat{\rho}=0.16$ \\
\hline
\end{tabular}


728

729

730

731

732

733

734

735

736

737

738

739

740

741

742

743

744

745

746

747

748

749

750

751

752

753

754

755

756

757

758

759

760

761

762

\section{FIGURE CAPTIONS}

Figure 1. Experimental workflow. Main steps of the workflow: following the CTA experiments (1), foci images were acquired (2) and from each image three statistical descriptors were selected to represent distinct morphological features used in visual scoring (3). The foci datasets of the two carcinogens were analyzed by factorizing the joint probability distribution into three conditional distributions (5a), each one subsequently modeled in the class of extended linear mixed-effects models (5b). The final models including the concentration were selected according to the BIC value, then they were exploited to test the effect of carcinogen concentration on each image descriptors by likelihood ratio tests $(5 \mathrm{c})$.

Figure 2. Boxplots of the number of foci. Boxplots of the number of Type III foci per dish given the carcinogen concentration. In $\mathrm{A}$ the number of foci from $\mathrm{NiCl}_{2}$ dataset are shown, while in $\mathrm{B}$ the number of foci from B[a]P dataset are displayed. Empty circles in B represent candidate outliers.

Figure 3. The dependence relationships between descriptors and concentration. The descriptors (ED, BD, MD) are displayed in blue circles, while nodes for concentration of a carcinogen (C) and Petri dish, both conditioning variables, are shown in yellow. Details of dependencies are provided in section 2.4.1.

Figure 4. Plot of expected value functions for the Equivalent Diameter (ED) given the carcinogen concentration. In $\mathrm{C}$ and $\mathrm{H}$, expected value functions of $\mathrm{ED}$ in models $\mathrm{N} 1\left(\mathrm{NiCl}_{2}\right)$ and B1 $(\mathrm{B}[\mathrm{a}] \mathrm{P})$ are plotted given the concentration. The grey shaded area represents pointwise confidence limits, on the scale of the linear predictor. Examples of foci showing ED values that can be found in the extreme regions of the graph are displayed in the panels A, B, D and E for model $\mathrm{N} 1$, and in F, G, I and $\mathrm{J}$ for model B1. The boxes on the $\mathrm{x}$ axis represent conditioning values of the carcinogen concentration.

Figure 5. Plot of expected value functions of Median (MD) given the carcinogen concentration. In $\mathrm{B}$ and $\mathrm{F}$ expected values of $\mathrm{MD}$ in model $\mathrm{N} 2\left(\mathrm{NiCl}_{2}\right)$ and $\mathrm{B} 2(\mathrm{~B}[\mathrm{a}] \mathrm{P})$, respectively are plotted given the concentration. The grey shaded area represents pointwise confidence limits, on the scale of the linear predictor. Examples of foci showing MD values corresponding to the area inside the blue circles are displayed in panels A, C and D, while examples of foci showing MD values corresponding to the area inside the red circles are displayed in panels $\mathrm{E}$ and $\mathrm{G}$. The boxes on the $\mathrm{x}$ axis represent conditioning values of carcinogen concentration. 
763

764

765

766

767

768

769

770

771

772

773

774

775

776

777

778

779

780

781

782

783

784

785

786

787

788

789

790

791

792

793

794

795

796

797

Figure 6. Plot of expected value functions of Boundary Index (BD) given the carcinogen concentration. In $\mathrm{B}$ and $\mathrm{E}$, expected values of $\mathrm{BD}$ in model $\mathrm{N} 3\left(\mathrm{NiCl}_{2}\right)$ and $\mathrm{B} 3(\mathrm{~B}[\mathrm{a}] \mathrm{P})$, respectively are plotted given the concentration. The grey shaded area represents pointwise confidence limits, on the scale of the linear predictor. Examples of foci showing BD values corresponding to the area inside the blue circles are displayed in panels $\mathrm{A}$ and $\mathrm{C}$, while $\mathrm{D}$ and $\mathrm{F}$ show foci having BD values corresponding to the area inside the red circles. The boxes on the $\mathrm{x}$ axis represent conditioning values of carcinogen concentration.

\section{SUPPORTING INFORMATION}

Figure S1. Boxplots of the number of foci before performing segmentation. Boxplots of the number of Type III foci per dish (y axes) before segmentation process and carcinogen concentrations (x axes). In A foci images from $\mathrm{NiCl}_{2}$ database are considered, while in $\mathrm{B}$ foci images from $\mathrm{B}[\mathrm{a}] \mathrm{P}$ database are included. Empty circles represent candidate outliers.

Figure S2. Diagnostics and additional plots of models of $\mathbf{N i C l}_{\mathbf{2}}$ dataset. Graphs are grouped according to model, by column: Model N1 (ED as the response variable), Model N2 (MD as the response variable) and Model N3 (BD as the response variable). In the first two rows diagnostic graphs are shown, quantile-quantile plots $(\mathrm{A}, \mathrm{B}, \mathrm{C})$ and plot of fitted values versus residuals $(\mathrm{D}, \mathrm{E}$, F), respectively. In G and I are plotted fitted values (Model N1) of the descriptor ED compared, respectively, to the linear predictor MD and to the linear predictor BD. The bars on the $\mathrm{x}$ axis represent $\mathrm{MD}$ and $\mathrm{BD}$ actual values, in plot $\mathrm{C}$ and $\mathrm{D}$, respectively. In $\mathrm{H}$ are plotted fitted values (Model N2) of the descriptor MD compared to the linear predictor MD (bars on the $\mathrm{x}$ axis represent BD actual values).

Figure S3. Diagnostics and additional plots of models of $\mathbf{B}[\mathbf{a}] \mathbf{P}$ dataset. Graphs are grouped according to model, by column: Model B1 (ED as the response variable), Model B2 (MD as the response variable) and Model B3 (BD as the response variable). In the first two rows diagnostic graphs are shown, quantile-quantile plots $(\mathrm{A}, \mathrm{B}, \mathrm{C})$ and plot of fitted values versus residuals $(\mathrm{D}, \mathrm{E}$, F), respectively. In G and I are plotted fitted values (Model B1) of the descriptor ED compared, respectively, to the linear predictor $\mathrm{MD}$ and to the linear predictor $\mathrm{BD}$. The bars on the $\mathrm{x}$ axis represent $\mathrm{MD}$ and $\mathrm{BD}$ actual values, in plot $\mathrm{C}$ and $\mathrm{D}$, respectively. In $\mathrm{H}$ are plotted fitted values (Model B2) of the descriptor MD compared to the linear predictor MD (bars on the $\mathrm{x}$ axis represent 


1
2
3
4
5
6
7
8
9
10
11
12
13
14
15
16
17
18
19
20
21
22
23
24
25
26
27
28
29
30
31
32
33
34
35
36
37
38
39
40
41
42
43
44
45
46
47
48
49
50
51
52
53
55
56

798 BD actual values).

799

800 Report S4. Supplementary statistical details and $\mathbf{R}$ code.

http://mc.manuscriptcentral.com/apptox 


\section{Cell Transformation Assay}

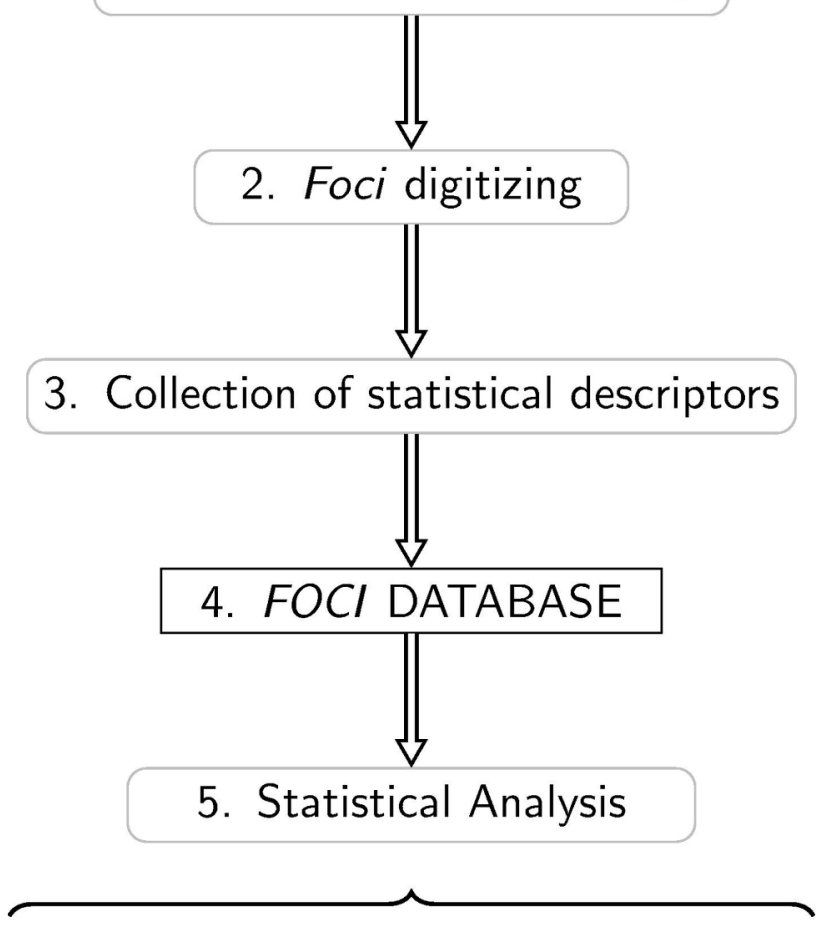

5b. An extended linear model developed for each descriptor

\section{5c. Hypothesis tests on the effect of chemical concentration}

Figure 1. Experimental workflow. Main steps of the workflow: following the CTA experiments (1), foci images were acquired (2) and from each image three statistical descriptors were selected to represent distinct morphological features used in visual scoring (3). The foci datasets of the two carcinogens were analyzed by factorizing the joint probability distribution into three conditional distributions (5a), each one subsequently modeled in the class of extended linear mixed-effects models (5b). The final models including the concentration were selected according to the BIC value, then they were exploited to test the effect of carcinogen concentration on each image descriptors by likelihood ratio tests (5c).

$$
116 \times 225 \mathrm{~mm}(300 \times 300 \mathrm{DPI})
$$


Number of foci for each $\mathrm{NiCl}_{2}$ concentration

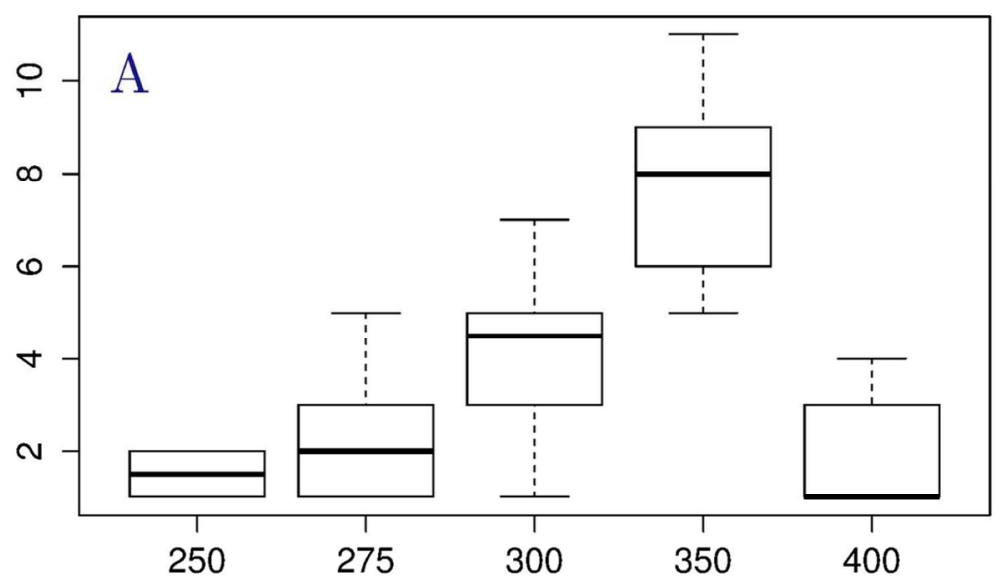

Number of foci for each $\mathrm{B}[\mathrm{a}] \mathrm{P}$ concentration

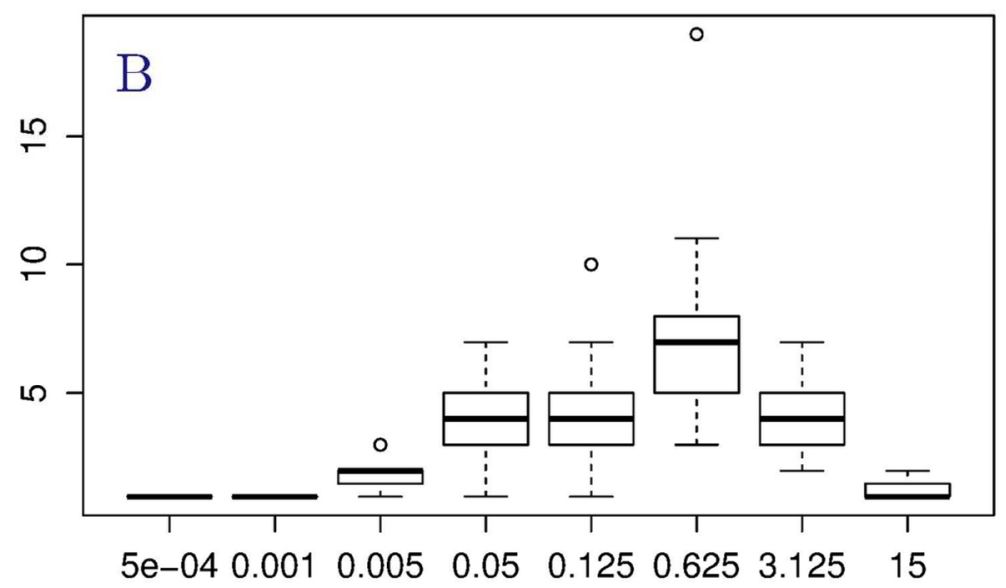

Figure 2. Boxplots of the number of foci. Boxplots of the number of Type III foci per dish given the carcinogen concentration. In $A$ the number of foci from $\mathrm{NiCl}_{2}$ dataset are shown, while in $\mathrm{B}$ the number of foci from $\mathrm{B}[\mathrm{a}] \mathrm{P}$ dataset are displayed. Empty circles in $\mathrm{B}$ represent candidate outliers.

$98 \times 134 \mathrm{~mm}(300 \times 300 \mathrm{DPI})$ 
Figure 3. The dependence relationships between descriptors and concentration. The descriptors (ED, BD, MD) are displayed in blue circles, while nodes for concentration of a carcinogen (C) and Petri dish, both conditioning variables, are shown in yellow. Details of dependencies are provided in section 3.1.

\section{$52 \times 35 \mathrm{~mm}(300 \times 300$ DPI $)$}



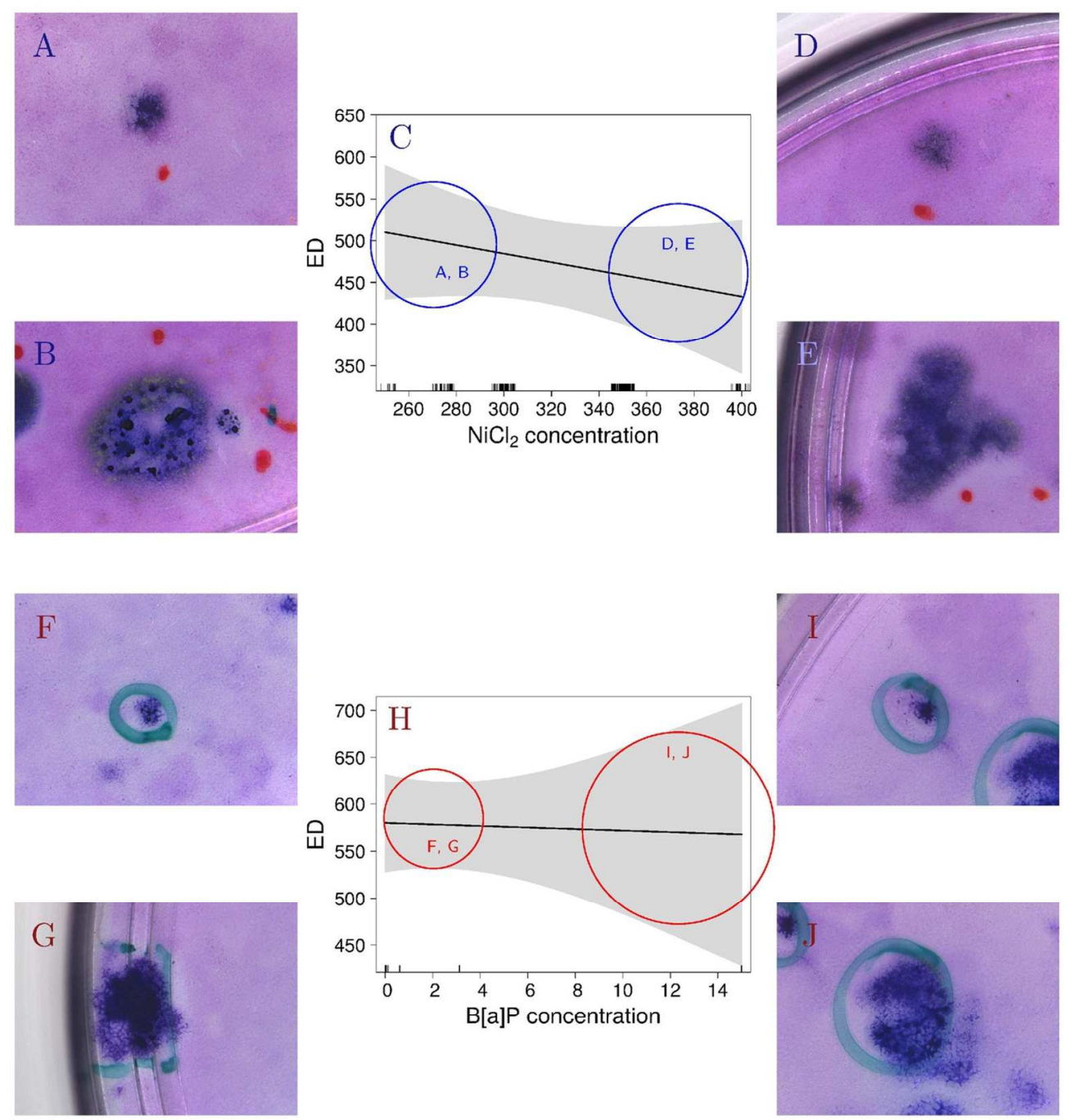

Figure 4. Plot of expected value functions for the Equivalent Diameter (ED) given the carcinogen concentration. In $\mathrm{C}$ and $\mathrm{H}$, expected value functions of $\mathrm{ED}$ in models $\mathrm{N} 1\left(\mathrm{NiCl}_{2}\right)$ and $\left.\mathrm{B} 1 \mathrm{~B}[\mathrm{a}] \mathrm{P}\right)$ are plotted given the concentration. The grey shaded area represents pointwise confidence limits, on the scale of the linear predictor. Examples of foci showing ED values that can be found in the extreme regions of the graph are displayed in the panels $A, B, D$ and $E$ for model $N 1$, and in F, G, I and J for model B1. The boxes on the $x$ axis represent conditioning values of the carcinogen concentration.

$$
119 \times 125 \mathrm{~mm}(300 \times 300 \text { DPI) }
$$



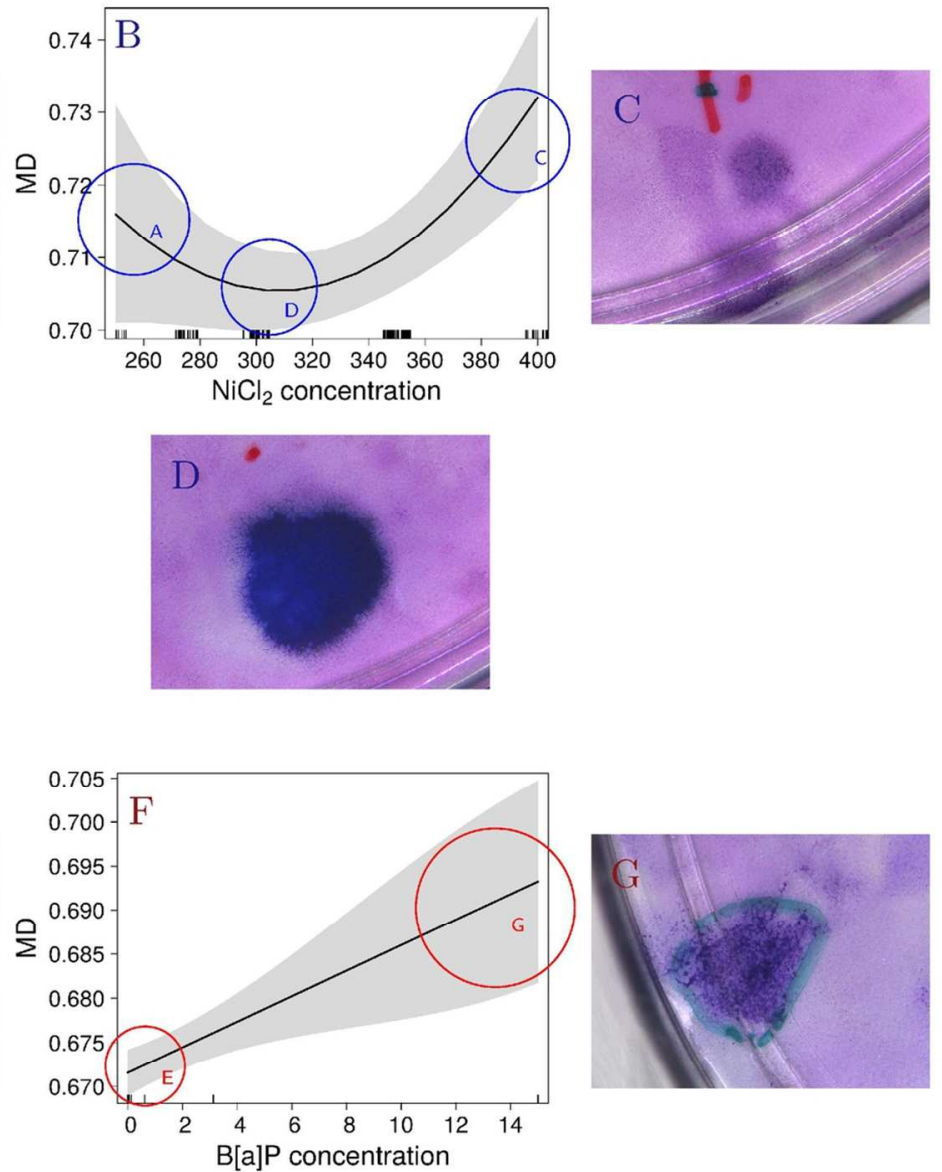

Figure 5. Plot of expected value functions of Median (MD) given the carcinogen concentration. In $B$ and $F$ expected values of $\mathrm{MD}$ in model $\mathrm{N} 2\left(\mathrm{NiCl}_{2}\right)$ and $\mathrm{B} 2(\mathrm{~B}[\mathrm{a}] \mathrm{P})$, respectively are plotted given the concentration. The grey shaded area represents pointwise confidence limits, on the scale of the linear predictor. Examples of foci showing MD values corresponding to the area inside the blue circles are displayed in panels $A, C$ and $\mathrm{D}$, while examples of foci showing MD values corresponding to the area inside the red circles are displayed in panels $\mathrm{E}$ and $\mathrm{G}$. The boxes on the $\mathrm{x}$ axis represent conditioning values of carcinogen concentration.

$109 \times 103 \mathrm{~mm}(300 \times 300 \mathrm{DPI})$ 

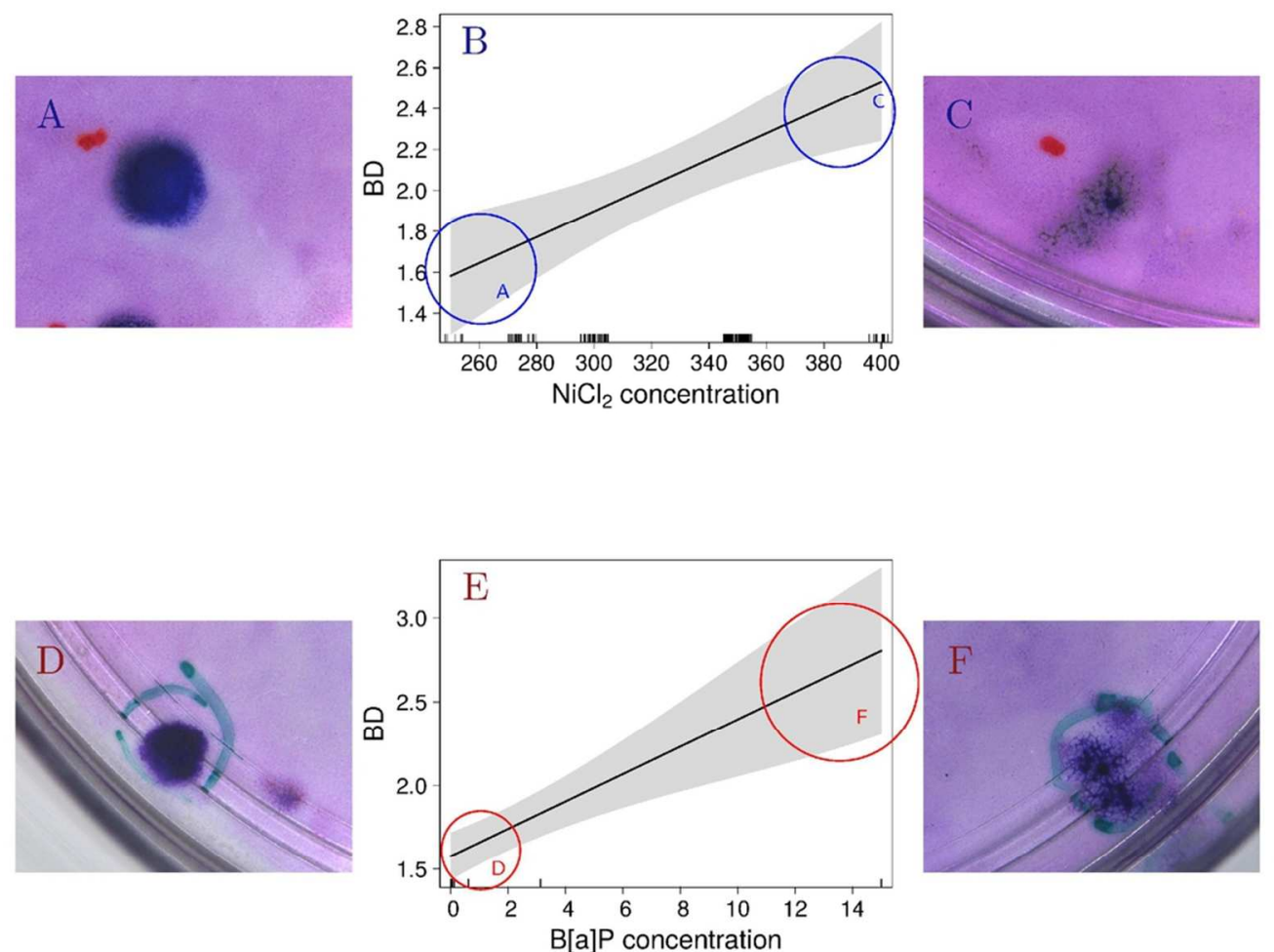

Figure 6. Plot of expected value functions of Boundary Index (BD) given the carcinogen concentration. In $B$ and $\mathrm{E}$, expected values of $\mathrm{BD}$ in model $\mathrm{N} 3\left(\mathrm{NiCl}_{2}\right)$ and $\mathrm{B} 3(\mathrm{~B}[\mathrm{a}] \mathrm{P})$, respectively are plotted given the concentration. The grey shaded area represents pointwise confidence limits, on the scale of the linear predictor. Examples of foci showing $\mathrm{BD}$ values corresponding to the area inside the blue circles are displayed in panels $A$ and $C$, while $D$ and $F$ show foci having $B D$ values corresponding to the area inside the red circles. The boxes on the $x$ axis represent conditioning values of carcinogen concentration.

$90 \times 72 \mathrm{~mm}(300 \times 300$ DPI $)$ 
1

2

3

4

5

6

7

8

9

10

11

12

13

14

15

16

17

18

19

20

21

22

23

24

25

26

27

28

29

30

31

32

33

34

35

36

37

38

39

40

41

42

4
4
4
4
4
4
4
5
5
5

4
4
4
4
4
4
5
5
5
5
5
5

5
5
5

5

5
5
5
6

5
6
Number of foci for each $\mathrm{NiCl}_{2}$ concentration $A$

Number of foci for each B[a]P concentration

B

B

57
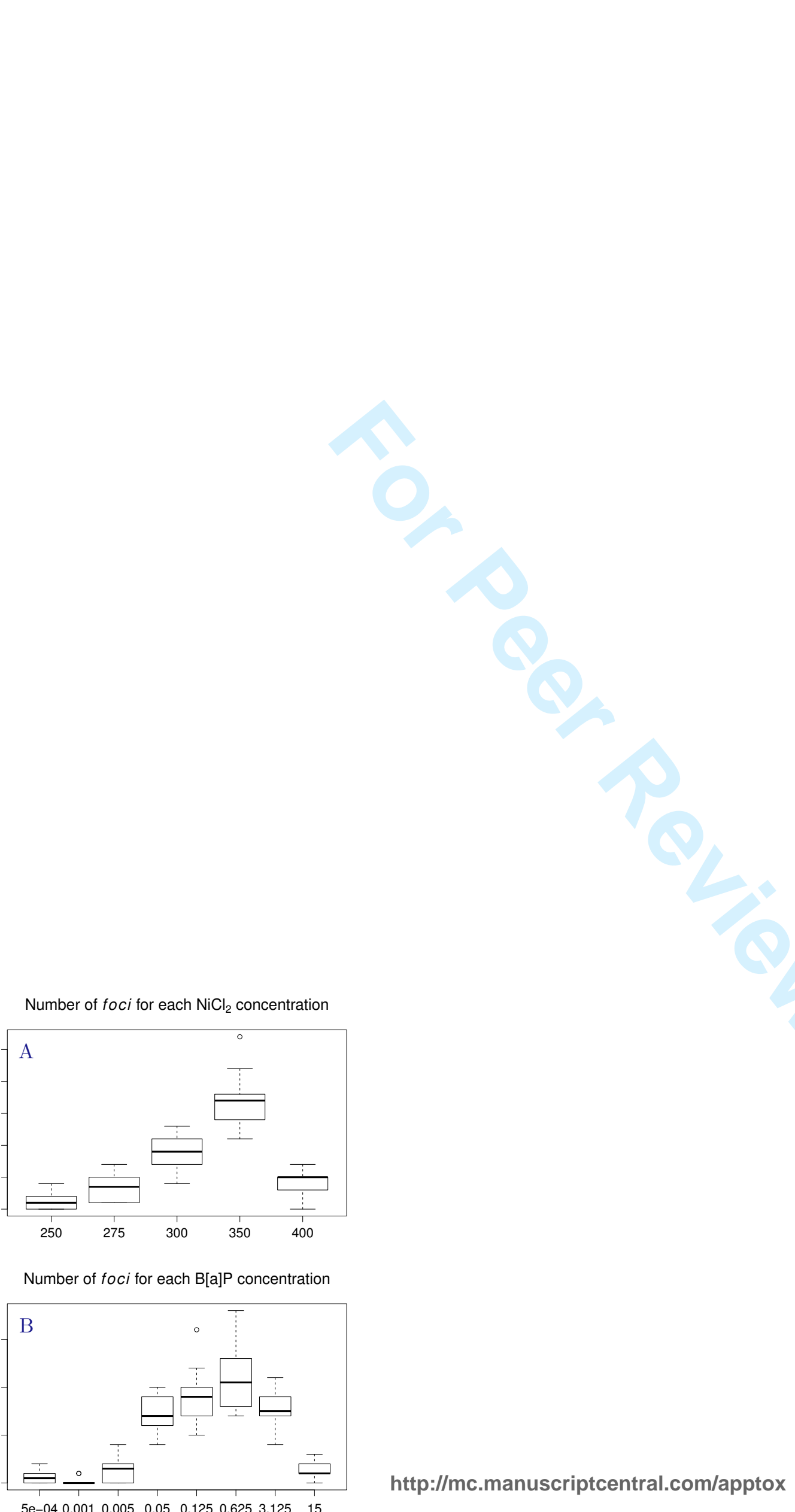
1

2

3

4

5

6
7

8

9

10

11

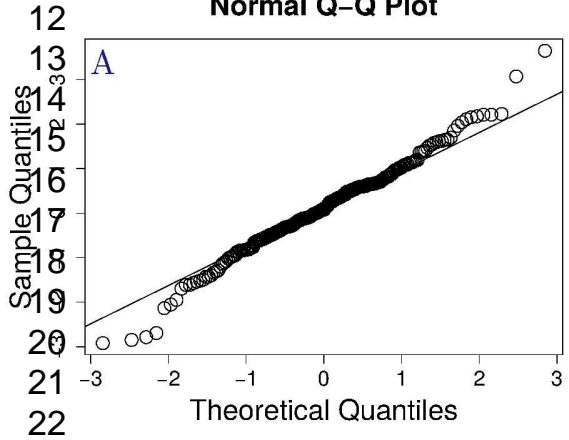

23

24

25

26

2
$\frac{62}{9}$
$\frac{9}{30}$
$\frac{3}{63}$

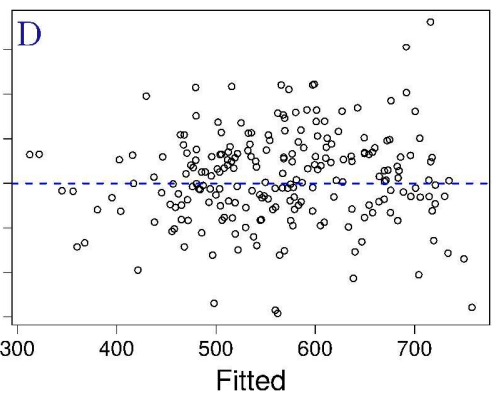

37

38

39

4
4
4
4

480
43
45
47
480

47

48

$4 \varsigma$

50

51

52

53

5
5
5
5

5
5
5

5

3

60

450

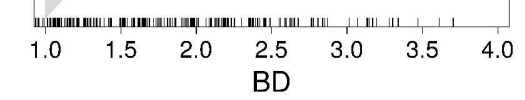

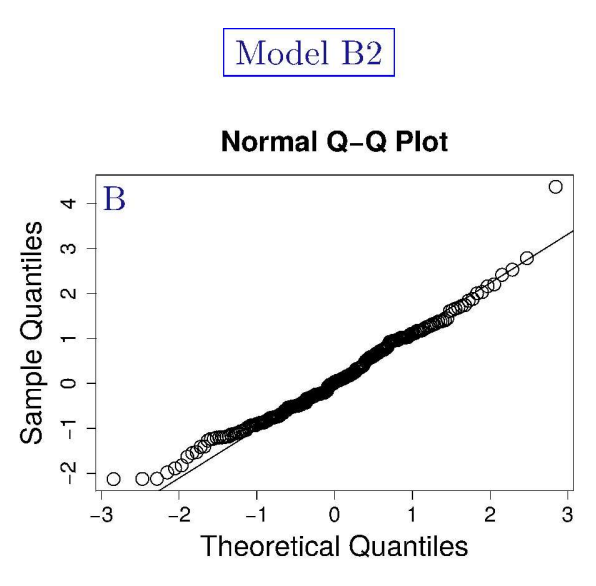
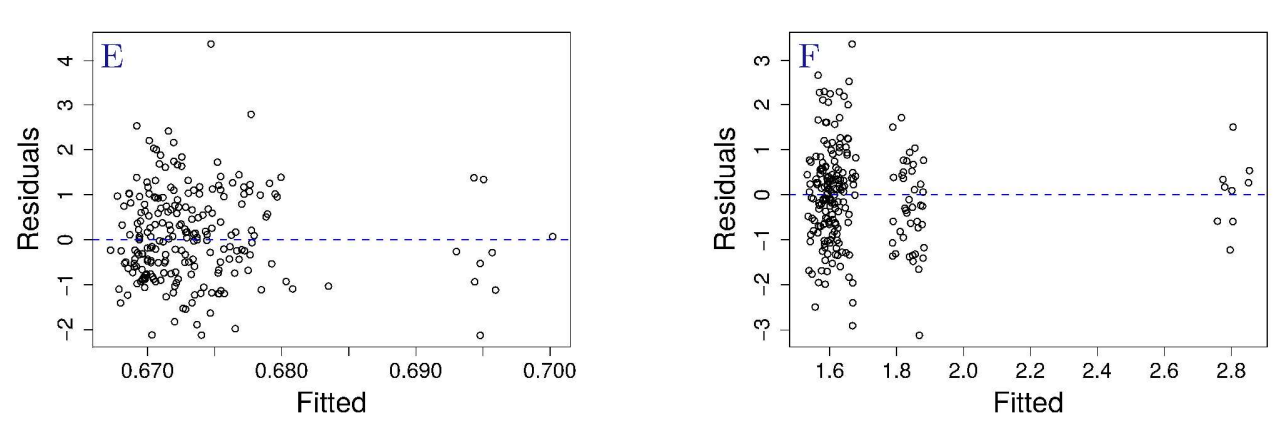

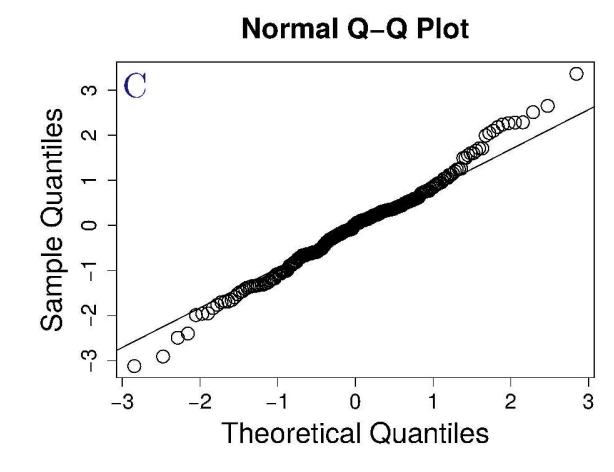

Model B3

Normal Q-Q Plot

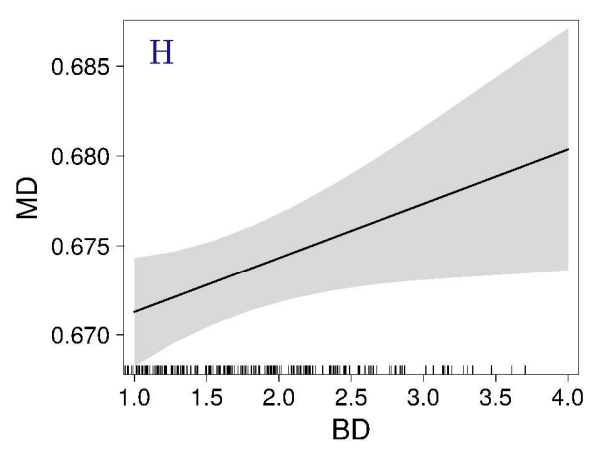




\section{Supporting Material S4}

This document is provided as supporting material for the paper "Relationship between increasing concentrations of a genotoxic and a non-genotoxic carcinogen and morphology in the Cell Transformation Assay as described by statistical image descriptors".

In the following sections R code for the statistical analysis performed in the main article is detailed, as well as supplementary materials of the presented models.

First, the following packages have to be loaded:

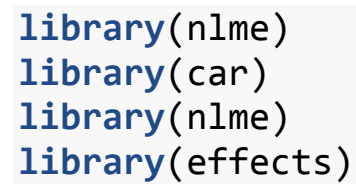

First, models for each descriptor as a response variable are fitted with generalized least squares accounting for unequal variance, and including a correlation structure (object of class ).

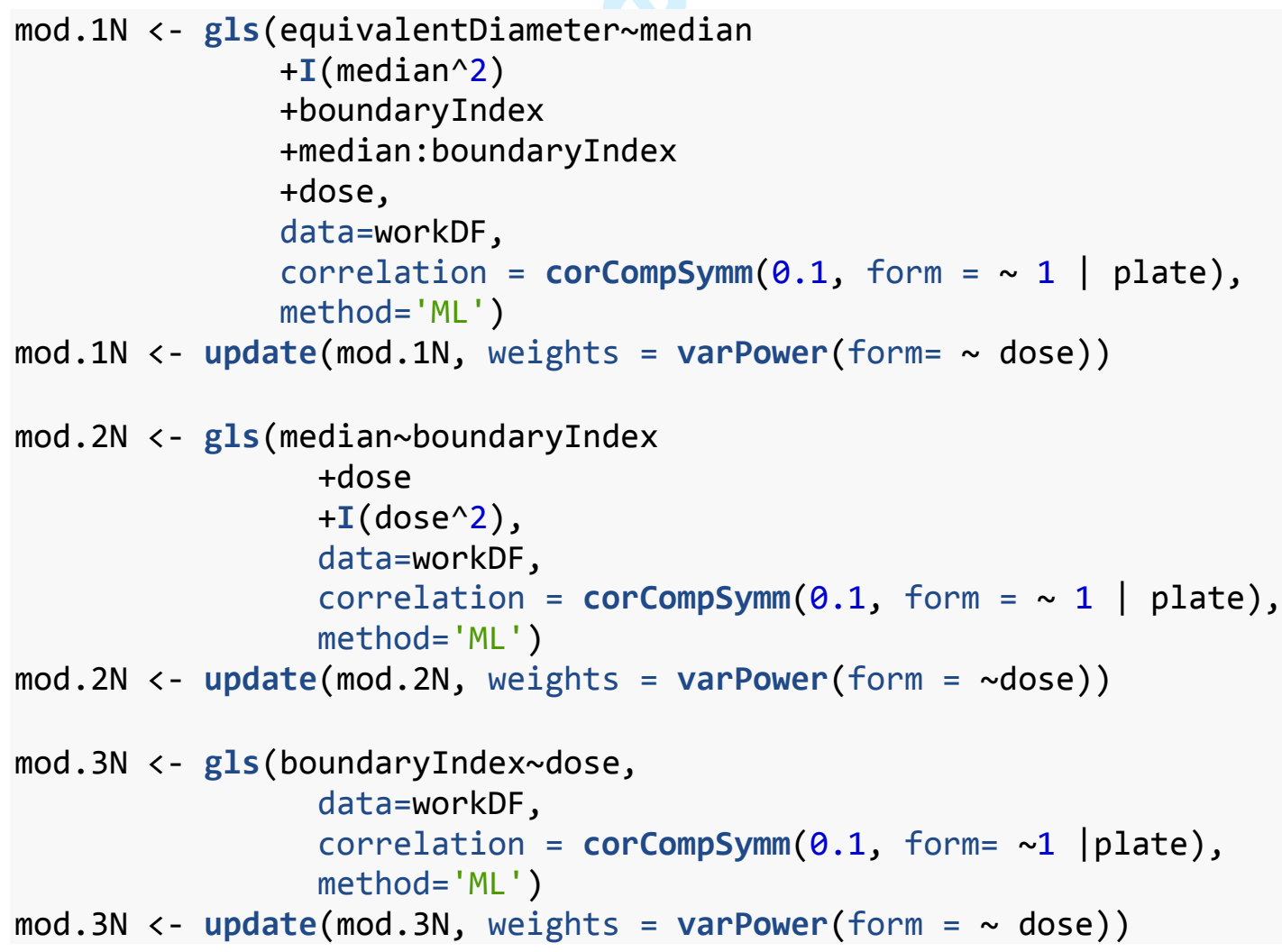

Then contribution of concentration on the expected value of descriptors was tested with reducted models: 
mod.1N_r <- update (mod.1N, . -dose)

anova (mod.1N, mod.1N_r)

mod.2N_r <- update $(\bmod .2 \mathrm{~N}, \sim$. -dose - I (dose^2))

anova (mod.2N, mod.2N_r)

mod.3N_r <- update $(\bmod .3 \mathrm{~N}, \sim$. -dose $)$

anova (mod.3N, mod.3N_r)

Diagnostic graphs were created with the following code (mod.1N shown as example), and are provided in Figure S2.

myres <- residuals (mod.1N, type='normalized')

qqnorm(myres)

qqline(myres)

plot (myres jitter (fitted(mod.1N), amount=.05), data=workDF)

abline $(h=0, \operatorname{col}=4,1 w d=2,1 t y=2)$

We here provide, as supporting materials, confidence intervals for estimated parameters and variance function for each fitted model:

intervals $(\bmod .1 \mathrm{~N})$

\#\# Approximate $95 \%$ confidence intervals

\#\#

\#\# Coefficients:

\#\# lower est. upper

\#\# (Intercept) $\quad 34516.6646 \quad 51993.6151 \quad 69470.56561$

\#\# median

\#\# I(median^2)

$-192036.6855-141843.6447-91650.60389$

\#\# boundaryIndex

$61201.4529 \quad 97223.6306 \quad 133245.80831$

\#\# doseNORM

$609.1660 \quad 1437.8238 \quad 2266.48156$

\#\# median:boundaryIndex

$-290.7646-108.0184$

74.72777

\#\# attr(,"label")

$\begin{array}{lll}-2990.1239 & -1848.8325 & -707.54106\end{array}$

\#\# [1] "Coefficients:"

\#\#

\#\# Correlation structure:

\#\# lower est. upper

\#\# Rho $0.03874005 \quad 0.1541243 \quad 0.3232199$

\#\# attr(,"label")

\#\# [1] "Correlation structure:"

\#\#

\#\# Variance function:

\#\# lower est. upper

$\begin{array}{lllll}\text { \#\# power } 0.01743034 & 0.4164543 & 0.8154782\end{array}$

\#\# attr(,"label")

\#\# [1] "Variance function:"

\#\#

\#\# Residual standard error: 


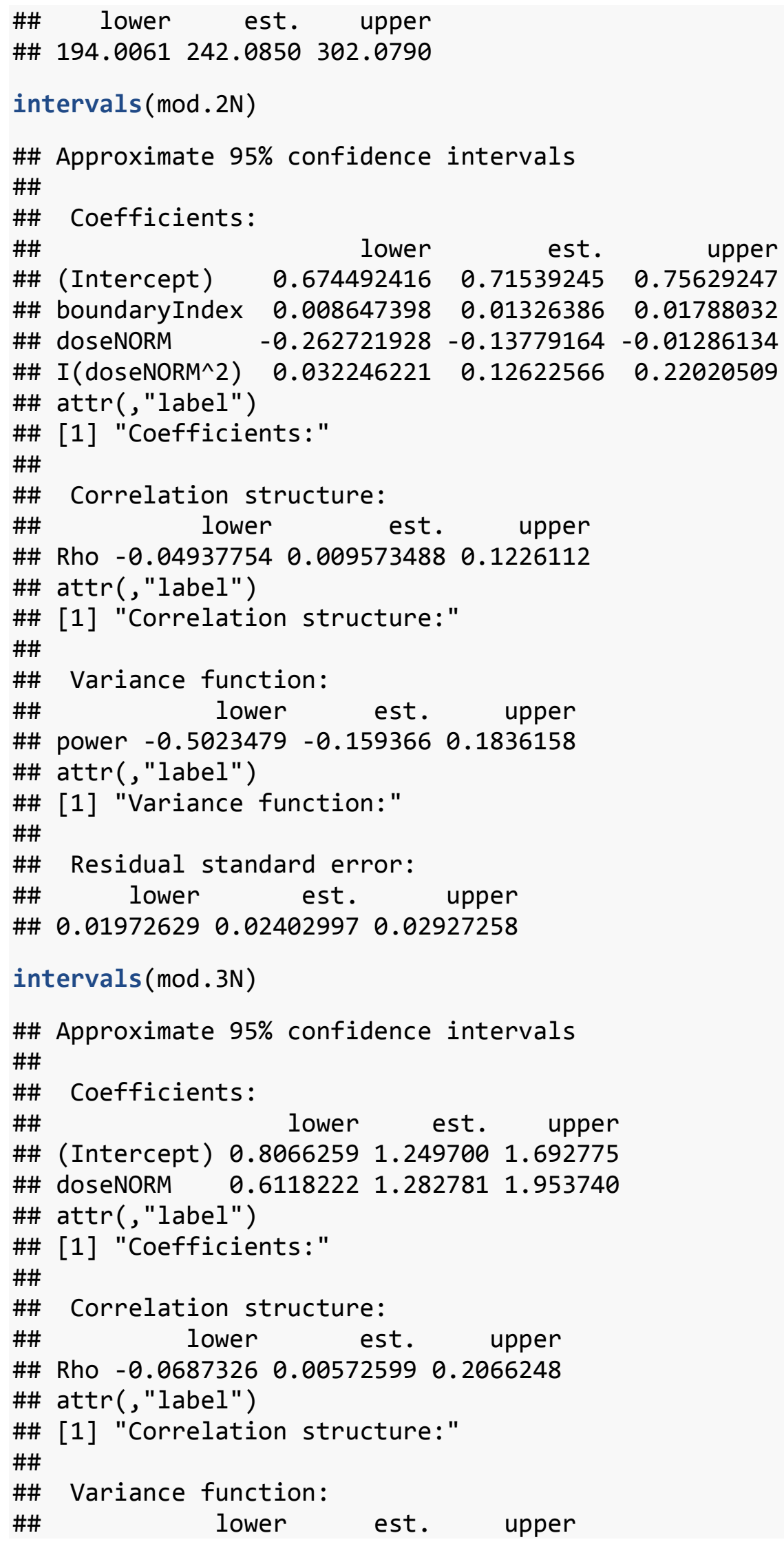




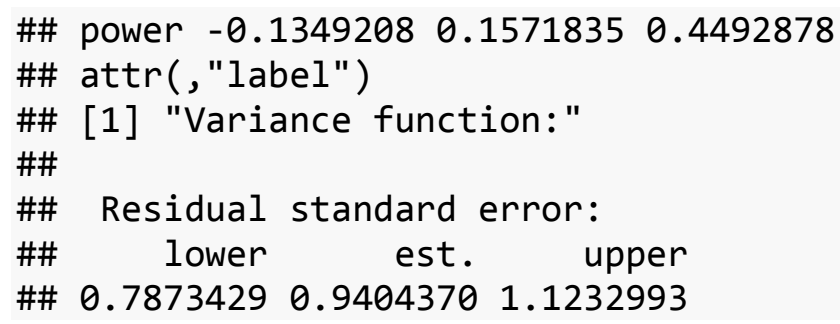

Plots of fitted values of each descriptor as response variable for each model and concentration variable was obtained with the following code (mod.1N shown as example):

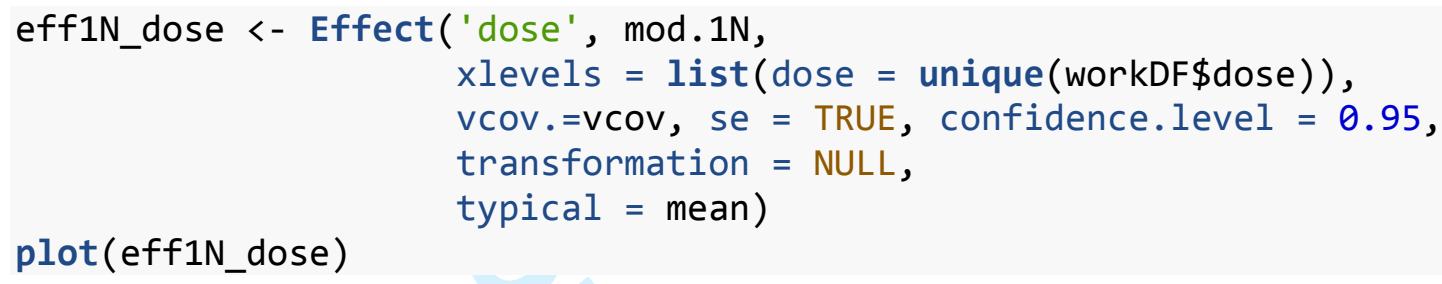

First, models for each descriptor as a response variable are fitted with generalized least squares accounting for unequal variance, and including a correlation structure (object of class ).

mod.1B <- gls(equivalentDiameter median

+boundaryIndex

$+\mathrm{I}$ (boundaryIndex^2)

+ dose, data=workDF, correlation $=\operatorname{corCompSymm}(0.1$, form $=\sim 1 \mid$ plate $)$, method= 'ML' )

$\bmod .1 \mathrm{~B}<-$ update $(\bmod .1 \mathrm{~B}$, weights $=\operatorname{varPower}($ form $=\sim$ dose $))$

$\bmod .2 \mathrm{~B}<-$ gls(median boundaryIndex

+dose,

data=workDF, correlation $=\operatorname{corCompSymm}(0.1$, form $=\sim 1 \mid$ plate $)$, method=' $M L$ ' )

$\bmod .2 \mathrm{~B}<-\operatorname{update}(\bmod .2 \mathrm{~B}$, weights $=\operatorname{varPower}($ form $=\sim$ dose $))$

mod.3B <- gls(boundaryIndex dose, data=workDF, correlation $=\operatorname{corCompSymm}(0.1$, form= 1 |plate $)$, method= 'ML ' )

$\bmod .3 \mathrm{~B}<-$ update $(\bmod .3 \mathrm{~B}$, weights $=\operatorname{varPower}($ form $=\sim$ dose $))$

Then contribution of concentration was tested with reducted models:

$\bmod .1 B \_r<-$ update $(\bmod .1 B, \sim .-$ dose $)$

anova (mod.1B, mod.1B_r)

mod.2B_r <- update (mod.2B, .-dose)

anova(mod.2B, mod.2B_r) 
$\bmod .3 B \_r<-$ update $(\bmod .3 B, \sim$. -dose $)$

anova (mod.3B, mod.3B_r)

Diagnostic graphs were created with the following code (mod.1B shown as example), and are provided in Figure S3.

myres <- residuals (mod.1B, type='normalized')

qqnorm(myres, main='q-q plot mod.1B')

qqline(myres)

plot (myres jitter (workDF\$doseSTD, amount=.05), data=workDF)

abline $(h=0, c o l=4$, lwd=2, lty $=2)$

We here provide, as supporting materials, confidence intervals for estimated parameters and variance function for each fitted model:

intervals $(\bmod .1 \mathrm{~B})$

\#\# Approximate $95 \%$ confidence intervals

\#\#

\#\# Coefficients:

\#\# lower est. upper

\#\# (Intercept) $\quad 1898.17101 \quad 3232.7439192 \quad 4567.316824$

\#\# median $\quad-6406.81328-4405.3403410-2403.867401$

$\begin{array}{llll}\text { \#\# boundaryIndex } & 93.19703 & 247.3053228 & 401.413611\end{array}$

$\begin{array}{llll}\text { \#\# I(boundaryIndex^2) } & -72.20875 & -38.6749767 & -5.141206\end{array}$

$\begin{array}{llll}\text { \#\# dose } & -11.28108 & -0.8103561 & 9.660365\end{array}$

\#\# $\operatorname{attr}($, ,label")

\#\# [1] "Coefficients:"

\#\#

\#\# Correlation structure:

\#\# lower est. upper

$\begin{array}{lllll}\text { \#\# Rho } 0.03737678 & 0.1450291 & 0.3018267\end{array}$

\#\# attr(,"label")

\#\# [1] "Correlation structure:"

\#\#

\#\# Variance function:

\#\# lower est. upper

\#\# power $-0.1072438-0.05933182-0.01141989$

\#\# attr(,"label")

\#\# [1] "Variance function:"

\#\#

\#\# Residual standard error:

\#\# lower est. upper

\#\# 203.7128228 .6996256 .7512

intervals (mod.2B)

\#\# Approximate 95\% confidence intervals

\#\# 
\#\# Coefficients:

\#\# lower est. upper

\#\# (Intercept) $\quad 0.6611820055 \quad 0.6662150440 .671248082$

$\begin{array}{llllll}\text { \# boundaryIndex } & 0.0002813363 & 0.003027738 & 0.005774139\end{array}$

\#\# dose $\quad 0.00062164440 .0014452370 .002268830$

\#\# attr(,"label")

\#\# [1] "Coefficients:"

\#\#

\#\# Correlation structure:

\#\# lower est. upper

\#\# Rho -0.0070801170 .064254250 .1753773$

\#\# attr(,"label")

\#\# [1] "Correlation structure:"

\#\#

\#\# Variance function:

\#\# lower est. upper

\#\# power -0.015437310 .030722610 .07688253$

\#\# attr(,"label")

\#\# [1] "Variance function:"

\#\#

\#\# Residual standard error:

\#\# lower est. upper

$\begin{array}{lllll}\text { \#\# } 0.01459902 & 0.01628850 & 0.01817350\end{array}$

intervals (mod.3B)

\#\# Approximate $95 \%$ confidence intervals

\#\#

\#\# Coefficients:

\#\# lower est. upper

\#\# (Intercept) 1.447339401 .588281351 .7292233

\#\# dose $\quad 0.045951920 .077332590 .1087133$

\#\# attr(,"label")

\#\# [1] "Coefficients:"

\#\#

\#\# Correlation structure:

\#\# lower est. upper

\#\# Rho 0.054664330 .15779610 .3005583

\#\# $\operatorname{attr}($, "label")

\#\# [1] "Correlation structure:"

\#\#

\#\# Variance function:

\#\# lower est. upper

\#\# power $-0.2263946-0.064418090 .09755844$

\#\# attr(,"label")

\#\# [1] "Variance function:"

\#\#

\#\# Residual standard error:

\#\# lower est. upper

$\begin{array}{lllll}\text { \#\# } 0.6090554 & 0.7188179 & 0.8483614\end{array}$ 
Plots of fitted values of each descriptor as response variable for each model and concentration variable was obtained with the following code (mod.1B shown as example):

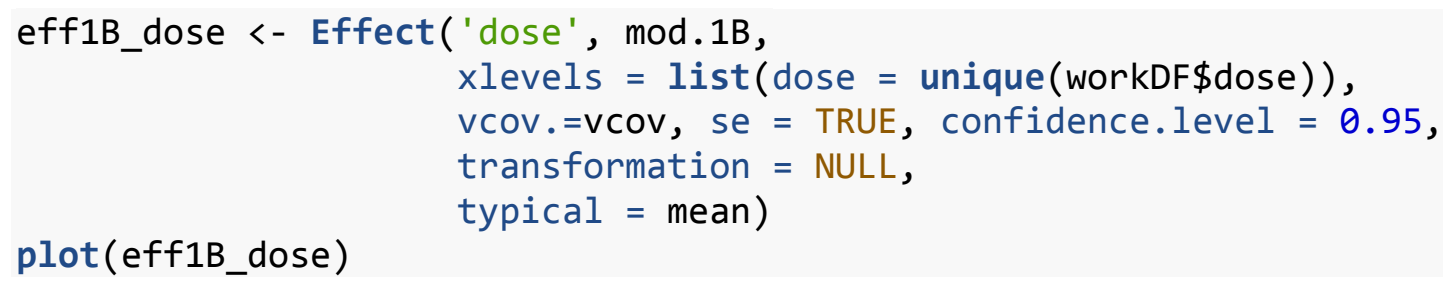

